Review Article

\title{
The State-of-the-Art Brief Review on Piezoelectric Energy Harvesting from Flow-Induced Vibration
}

\author{
Hongjun Zhu $\mathbb{D}^{1},{ }^{1}$ Tao Tang, ${ }^{1}$ Huohai Yang, ${ }^{1}$ Junlei Wang, ${ }^{2}$ Jinze Song, ${ }^{1}$ and Geng Peng ${ }^{3}$ \\ ${ }^{1}$ State Key Laboratory of Oil and Gas Reservoir Geology and Exploitation, Southwest Petroleum University, Chengdu 610500, \\ China \\ ${ }^{2}$ School of Mechanical and Power Engineering, Zhengzhou University, Zhengzhou 450000, China \\ ${ }^{3}$ Engineering Technology Research Institute, PetroChina Southwest Oil and Gasfield Company, Chengdu 610031, China
}

Correspondence should be addressed to Hongjun Zhu; zhuhj@swpu.edu.cn

Received 22 September 2020; Revised 20 October 2020; Accepted 20 March 2021; Published 1 April 2021

Academic Editor: Paola Forte

Copyright ( 2021 Hongjun Zhu et al. This is an open access article distributed under the Creative Commons Attribution License, which permits unrestricted use, distribution, and reproduction in any medium, provided the original work is properly cited.

\begin{abstract}
Flow-induced vibration (FIV) is concerned in a broad range of engineering applications due to its resultant fatigue damage to structures. Nevertheless, such fluid-structure coupling process continuously extracts the kinetic energy from ambient fluid flow, presenting the conversion potential from the mechanical energy to electricity. As the air and water flows are widely encountered in nature, piezoelectric energy harvesters show the advantages in small-scale utilization and self-powered instruments. This paper briefly reviewed the way of energy collection by piezoelectric energy harvesters and the various measures proposed in the literature, which enhance the structural vibration response and hence improve the energy harvesting efficiency. Methods such as irregularity and alteration of cross-section of bluff body, utilization of wake flow and interference, modification and rearrangement of cantilever beams, and introduction of magnetic force are discussed. Finally, some open questions and suggestions are proposed for the future investigation of such renewable energy harvesting mode.
\end{abstract}

\section{Introduction}

Over the past two decades, the demand for electrochemical batteries has been tremendously growing with the blooming of wireless sensor networks (WSNs) and microelectronicmechanical systems (MEMSs) [1]. Nevertheless, the power supply of such microelectric components from the combustion of fossil fuel not only causes the waste of energy but also pollutes the environment. Therefore, renewable and clean energy harvesting from ambient environment is increasingly attracting attention in the worldwide energy market [2-4]. Electricity could be converted from the mechanical energy of wind, tide, current, and other natural resources by considering electromagnetic, electrostatic, dielectric, triboelectric, and piezoelectric effects. The coil is usually used in an electromagnetic conductor to create electricity when there is a relative movement between the coil and the magnet. The generating capacity depends on the strength of the magnetic field, relative movement velocity and the turns of the coil [5]. The key component of an electrostatic generator is a variable capacitor, converting the energy of mechanical motion to electrical one through altering the capacitance [6]. The capacitor is also employed in dielectric generator, where the capacitor usually consists of two plates electrically isolated from each other by air, vacuum, or an insulator. Such kind of generator is suitable for distributed power systems and renewable energy storage [7] due to its ultrahigh power density, long lifetime, and high cycling stability [8]. A triboelectric generator utilizes the transfer of surface charge during the relative motion between two materials with different triboelectric polarities [9]. The piezoelectric effect is described as the asymmetric shift of charges or ions of piezoelectric materials when exposed to mechanical strain [10]. Piezoelectric effects can be classified into direct and converse piezoelectric effects, where the former one is based on the effect of applied pressure and later one is attained by shape deformation. Considering the directions of polarization and stress [11], there are two main 
operation modes for piezoelectric materials as illustrated in Figure $1 . d_{31}$ and $d_{33}$ represent two operation modes in which the electric field is perpendicular to and align with the direction of applied stress. The former one is commonly used in bending-beam structures because of its lower coupling coefficient. In contrast, the $d_{33}$ mode appears in direct compressive/tensile harvesters or harvesters with the interdigitated electrode. Through contrastive analysis, the piezoelectric effect is widely applied due to its large piezoelectric coefficients and electromechanical coupling factors as well as high energy convention rates [11, 12].

The premise of generating electricity is the piezoelectric material exerted mechanical strains. Thus, the deformation of piezoelectric material and its frequency is closely related to the electricity output. In the past few decades, a wide range of piezoelectric materials were investigated, including inorganic, organic, composite, and bioinspired piezoelectric materials. The characteristics and application of these four piezoelectric materials are compared in Table 1. For the first type of piezoelectric material (inorganic), perovskite, ilmenite, bismuth-layer, and tungsten bronze are the common structure ferroelectrics, and perovskite is the prominent piezoceramic crystal structure. As an organic piezoelectric material, the polyvinylidene fluoride (PVDF) polymer was first proposed by Kawai [13], having advantages of being flexible, lightweight, and low-cost [14]. The composite of inorganic and organic materials combines the advantages, presenting enough flexibility and piezoelectric coefficient. Therefore, it is preferred in commercial usage. Although the bioinspired material is a novel type proposed in recent years, its performance has not been well understood and hence it has not been used in practice.

Besides, the architecture configuration has a significant effect on the performance of piezoelectric materials. As listed in Table 2, the piezoelectric material is mounted in a generator in variable configurations. A cantilever beam attached with a piezoelectric layer and a tip mass is a superior arrangement due to the large deformation and high output power $[17,18]$. Furthermore, the working range of response frequency is broadened by replacing the tip mass with a magnetic mass.

As shown in Figure 2, a variety of available energy resources ranging either from building and bridge oscillation to human motion or from water flow to wind can be utilized to power small-scale devices such as monitors or pacemakers by the piezoelectric energy harvester. As one of the mechanical energy, structural vibration widely exists in our surroundings. An increasing number of scholars have been concerning the conversion of vibration energy to electric energy due to the considerable advantages such as high energy density and less dependence on weather [25-27]. Salazar et al. [28] pointed out that although the harvesting of vibration energy is not as mature as that from solar, wind, and hydropower energy, vibration energy harvester can fulfill the requirements from different scale, including macro-, micro-, or even nanoscale, with low-cost maintenance. The applicability of vibration energy harvesting has been confirmed in the railway environments, and several associated self-powered devices have been
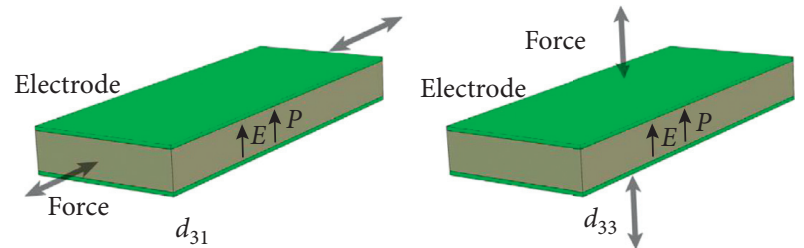

FIgURE 1: The $d_{31}$ and $d_{33}$ operation modes [11].

designed and tested [29]. The railway track usually vibrates in a low range of frequency with one dominant frequency [30], determining the power output. To enlarge the scope of application and improve the energy harvesting efficiency, both the linear and nonlinear vibration of energy generators have been extensively investigated in recent years [31-34].

The vortex-induced vibration aquatic clean energy (VIVACE, as seen in Figure 3) proposed by Bernitsas et al. [35] could convert the current kinetic energy into vibration energy by flow-induced vibration (FIV) and then further convert into electrical energy via an electromagnetic induction motor. FIV is a physical phenomenon in the engineering field caused by alternately shedding of vortices or dynamic instability [36]. Vortex-induced vibration (VIV) and galloping are two common types of FIV. The boundary layer separates from a bluff body when flow pasts it at a sufficient high $\operatorname{Re}(\operatorname{Re}=U D / v$, where $U$ is the free stream velocity, $D$ is the feature-length of the bluff body, and $v$ is the fluid kinematic viscosity), generating unsteady and periodical shedding vortices in the wake of the structure [37]. Consequently, fluctuating fluid forces are imposed on the bluff body and hence leading to vibration response, known as VIV. Galloping is usually caused by dynamic instability due to internal turbulence or other kinds of disturbance [38]. It is proved that the piezoelectric energy harvesting from the vibration energy contained in VIV and galloping is feasible with great potential [39-42].

Numerous methods have been proposed to enhance the harvested power of piezoelectric harvesters. Wherein, a commonly used method is tuning the structural frequency to match the resonant vibration frequency, yielding severer response and consequent higher power [43-45]. Moon et al. [46] tested the cantilever beam with a tuned proof mass and observed a $426.6 \%$ increase in bandwidth at the same output level and $508.5 \%$ growth in power as compared to the conventional form. Apart from the mass, the geometry of the cantilever plays an important role in tuning the resonant frequency. The rectangular column cantilevers are widely used because of their convenient installation and effectiveness in harvesting energy [47-49]. Cantilevers with other shapes of cross-section such as triangular [50], trapezoidal [51], and circular [52] are also evaluated.

The orientation of piezoelectric harvester affects energy harvesting, as reported in the experimental study by Dai et al. [53]. As shown in Figure 4, the vertical arrangement was observed to generate the largest power among the four typical configurations, mainly due to the smallest coupled damping. 
TABLE 1: Comparison of four typical piezoelectric materials.

\begin{tabular}{|c|c|c|c|c|}
\hline $\begin{array}{l}\text { Type of } \\
\text { piezoelectric }\end{array}$ & Typical material & Advantages/dis & advantages & Application \\
\hline Inorganic & $\begin{array}{c}\mathrm{BaTiO}_{3}(\mathrm{BT})[15] \\
\mathrm{PbTiO}_{3}(\mathrm{PT})\end{array}$ & $\begin{array}{c}\text { The first proposed piezoelectric } \\
\text { ceramic and high dielectric constant } \\
\text { High electromechanical coupling } \\
\text { property }\end{array}$ & $\begin{array}{l}\text { Commonly used in practice but } \\
\text { chip easily under sufficient }\end{array}$ & $\begin{array}{c}\text { Commercially } \\
\text { used }\end{array}$ \\
\hline & $\begin{array}{l}\text { Zirconate-lead titanate } \\
\text { system }(\mathrm{PZT})[16]\end{array}$ & Higher piezoelectric property & & \\
\hline Organic & $\begin{array}{l}\text { Polyvinylidene fluoride } \\
\text { polymer (PVDF) }\end{array}$ & $\begin{array}{l}\text { Low permittivity and acoustic } \\
\text { impedance }\end{array}$ & $\begin{array}{c}\text { Flexible, durable, and easy for } \\
\text { processing }\end{array}$ & $\begin{array}{l}\text { Commercially } \\
\text { used }\end{array}$ \\
\hline Composite & $\begin{array}{l}\text { Composites of PZT/ } \\
\text { PVDF }\end{array}$ & $\begin{array}{l}\text { Superior piezoelectric performance } \\
\text { and comparable flexibility }\end{array}$ & Flexibility, quick pack & $\begin{array}{l}\text { Commercially } \\
\text { used }\end{array}$ \\
\hline $\begin{array}{l}\text { Bioinspired } \\
\text { materials }\end{array}$ & $\begin{array}{l}\text { Eggshell, membrane, fish } \\
\text { swim, onion skin }\end{array}$ & $\begin{array}{l}\text { Nontoxic, biodegradable, } \\
\text { biocompatible }[10]\end{array}$ & $\begin{array}{l}\text { Effectively reduce the biologic } \\
\text { and toxic e-wastes }\end{array}$ & In developing \\
\hline
\end{tabular}

This work aims to give a brief review of the energy extraction technologies from flow-induced vibrations and the associated improvement methods of piezoelectric harvesters.

\section{Energy Harvesting from the Single Harvester}

2.1. Irregularity. After the proposal of VIVACE, Bernitsas and his colleagues $[54,55]$ found that the vibration response and energy harvesting efficiency could be further enhanced by symmetrically attaching a couple of strips on the front surface of a circular cylinder, called the passive turbulence control (PTC). The presence of strips altered the pressure distribution around the cylinder surface and tripped the boundary layer. Zhu et al. [56] numerically investigated the FIV of a locally rough cylinder with two symmetrical strips attached on its surface and examined the effects of location and shape of strips. The numerical results indicated that the gap between VIV and galloping was filled by placing strips in the front surface of the cylinder, which was illustrated from the changes in turbulent intensity, vortex shedding mode, and wake width. In a wind tunnel, $\mathrm{Hu}$ et al. [57] experimentally investigated the response of a circular cylinder with a pair of small-diameter cylindrical rods. It was observed that the vibration of the roughened cylinder was sensitive to the circumferential location. The control rods placed at $\theta=60^{\circ}(\theta$ denotes the circumferential location measured from the front stagnation point) significantly expanded the aeroelastic unstable range of the circular cylinder and hence presented a superiority over the plain circular cylinder in energy harvesting. Similarly, through a series of wind-tunnel experiments, Wang et al. [58] demonstrated $\theta=60^{\circ}$ was the optimal location of rectangular control rods. Hu et al. [59] compared the effects of three different cross-sectional control rods including circular, triangular, and square, as shown in Figure 5. The results illustrated that the attachment of triangular rods exhibited the best performance. $\mathrm{Hu}$ et al.
[60] also investigated the performance of energy harvesting by attaching fins to the corners of a square cylinder (Figure 6). It was proved that the attachment of fins to the leading edge attained a power 2.5 times than that harvested by a plain square cylinder. Song et al. [61] pointed out the splitter plate placed in the near wake of a circular cylinder could improve the performance of piezoelectric harvester. It was found that the length of $0.65 D$ ( $D$ is the diameter of circular cylinder) was the optimal length for enlarging the harvested power. Zhu et al. [62] evaluated the vibration response of a circular cylinder with splitter plates placed upstream and downstream individually and simultaneously. The galloping was observed to occur at $U_{r}>9\left(U_{r}\right.$ is the reduced velocity), and the longer the plate is, the larger the onset reduced velocity of galloping. This similar phenomenon was observed by Zhu and Liu [63] for a circular cylinder attached with a wavy plate.

Apart from the conventional shapes, Wang et al. [64] studied the performance of piezoelectric harvester with Y-shaped attachments, as shown in Figure 7(a). The transition from vortex-induced vibration into galloping was observed with the result of enhanced energy harvesting. Zhu et al. $[65,66]$ proposed a bioinspired fin-shaped strip with the intent of altering the vortex shedding as the swimming fish, as depicted in Figure 7(b). A pair of fin-shaped strips was attached to the front surface of a circular cylinder. It was reported that the time-mean drag and the lift fluctuation at $\mathrm{Re}=180$ were increased by $38.7 \%$ and $97.7 \%$, respectively, by placing a pair of $40^{\circ}$-fin-shaped strips at $\theta=60^{\circ}$. Ding et al. [67] examined the harvested power of a circular cylinder attached with such fin-shaped strips. The results indicated that the maximum harvested power could reach 25.5 times of the power generated by a smooth circular cylinder when the placement incoming angle is in the range of $30^{\circ}-60^{\circ}$.

Table 3 briefly summarizes the performance of piezoelectric harvester with attachments in the aforementioned literature. It is noted that the output power is lower than that 
TABLE 2: Types of architecture configuration for the piezoelectric harvester.

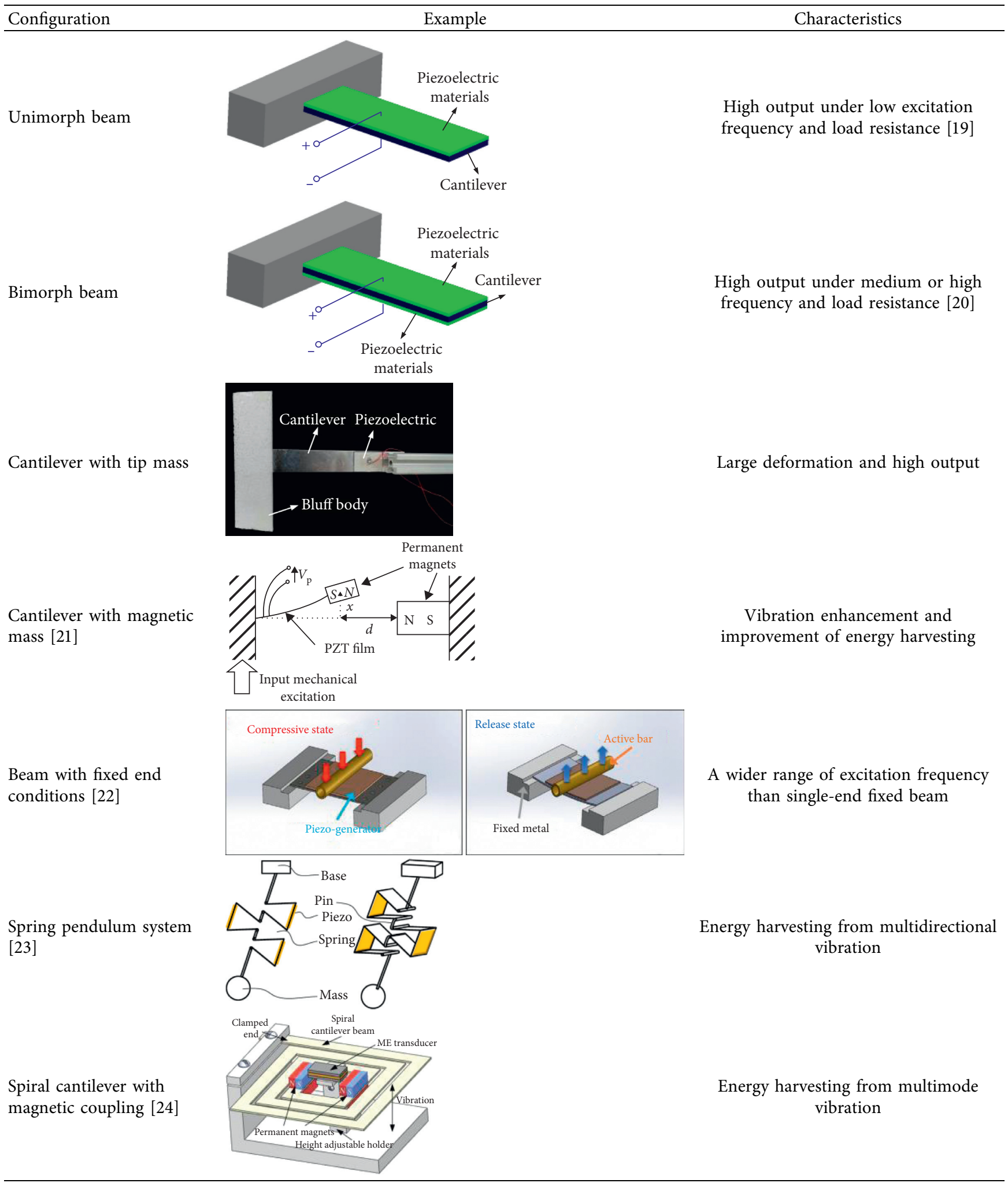

harvested by the VIVACE converter via the electromagnetic effect $[68,69]$. This implies that the microwatt-scale piezoelectric energy harvester is more suitable for powering small-scale devices. The shape of attachments has a significant impact on the performance of energy harvesting. Generally, the attachments with sharp corners are more likely to enhance the vibration, due mainly to the forward movement of boundary layer separation points [27, 70, 71]. 


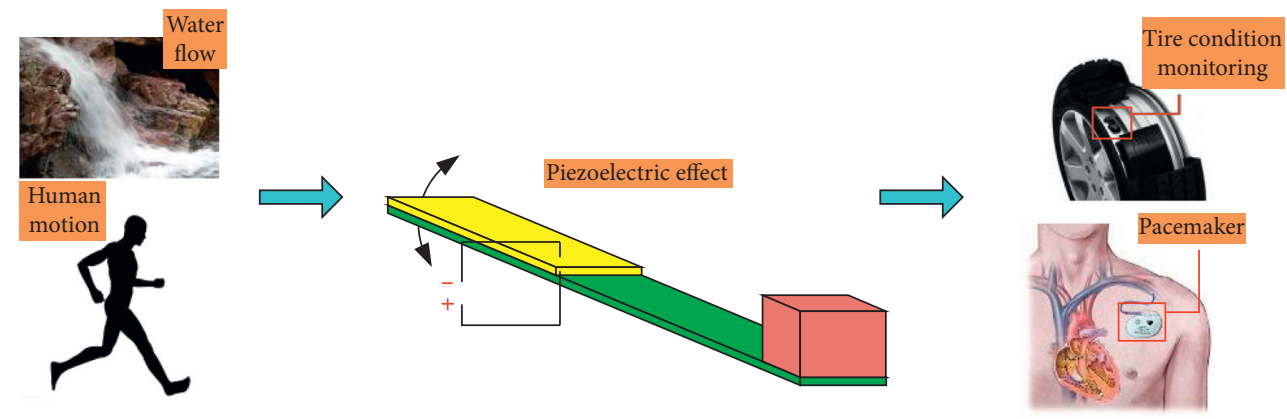

FIGURE 2: The typical resources for piezoelectric energy harvesting.

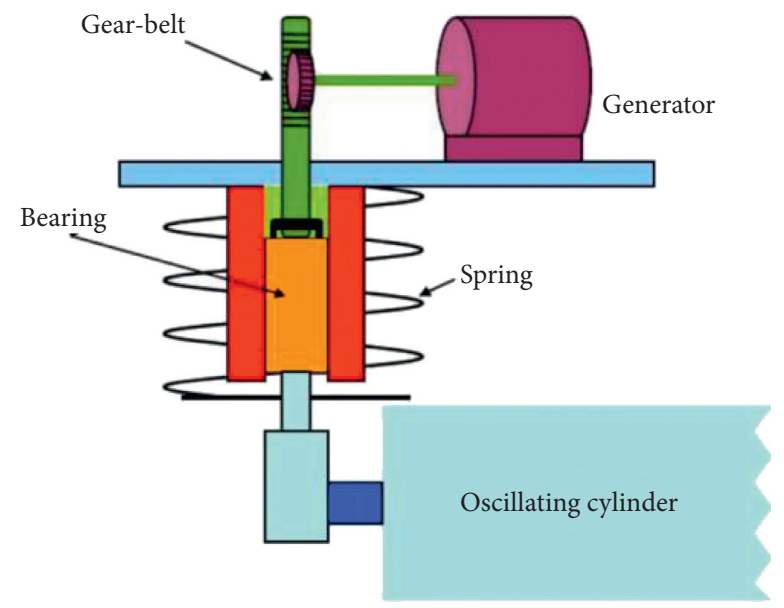

Figure 3: The VIVACE configuration proposed by Bernitsas et al. [35].

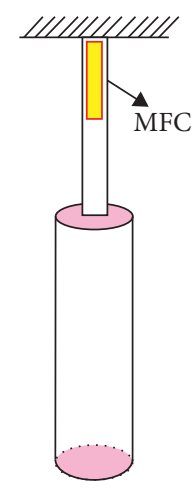

(a)

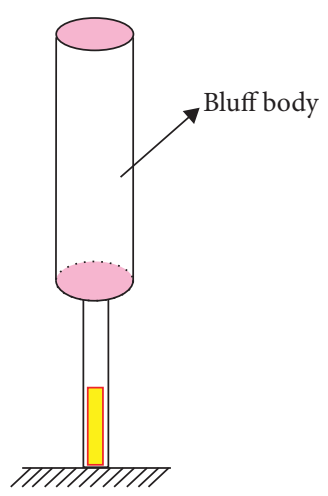

(b)

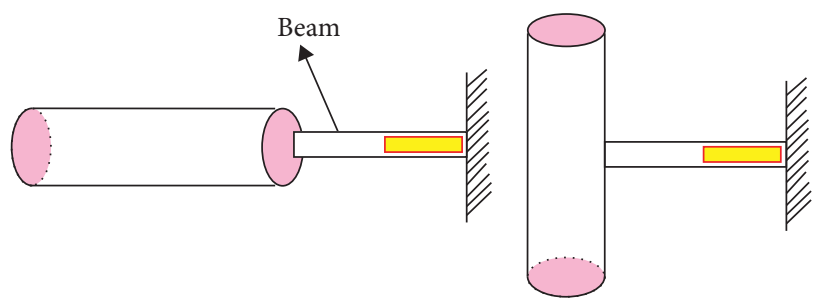

(c) (d)

Figure 4: Different orientations. (a) Bottom. (b) Top. (c) Horizontal. (d) Vertical [53].

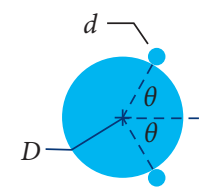

(a)
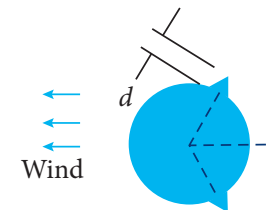

(b)

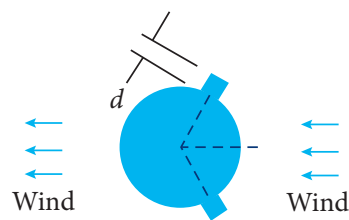

(c)

Figure 5: Cross-sectional shapes of control rods. (a) Circular. (b) Triangular. (c) Square [59]. 

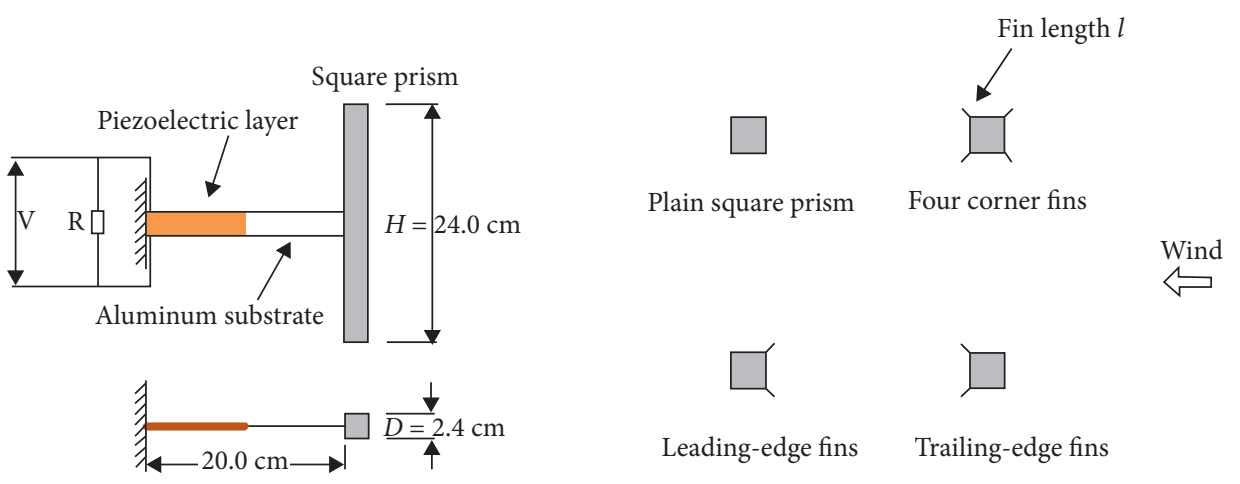

FIGURE 6: Sketch of the piezoelectric harvester with fins [60].

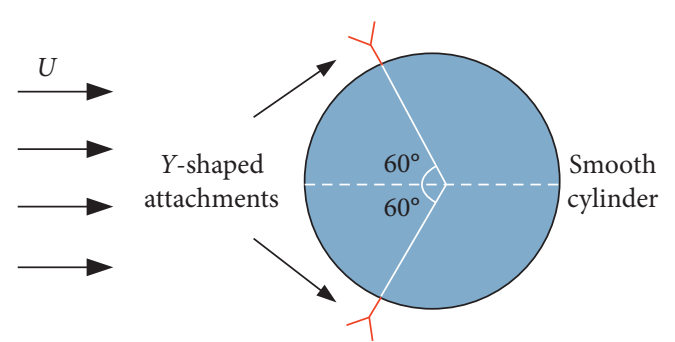

(a)

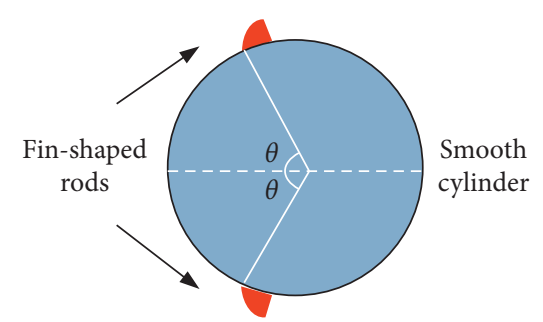

(b)

Figure 7: (a) Y-shaped attachments [64]. (b) Fin-shaped strips [65].

TABLE 3: Brief summary of reported studies on piezoelectric harvester using localized roughness.

\begin{tabular}{lcccccc}
\hline Researcher & Cylinder & Shape of control rods & Flow velocity & Load & Vibration type & Output power \\
\hline Hu et al. [60] & Square & Fin-shaped & $7.0 \mathrm{~m} / \mathrm{s}$ & $5 \mathrm{M} \Omega$ & Galloping & $39.6 \mu \mathrm{W}$ \\
Hu et al. [57] & Circular & Circular & $5.9 \mathrm{~m} / \mathrm{s}$ & $5 \mathrm{M} \Omega$ & VIV, galloping & $71.4 \mu \mathrm{W}$ \\
Hu et al. [59] & Circular & Circular & $6.4 \mathrm{~m} / \mathrm{s}$ & $5 \mathrm{M} \Omega$ & Galloping & $55.2 \mu \mathrm{W}$ \\
Hu et al. [59] & Circular & Triangular & $6.0 \mathrm{~m} / \mathrm{s}$ & $5 \mathrm{M} \Omega$ & Galloping & $54.2 \mu \mathrm{W}$ \\
Hu et al. [59] & Circular & Square & $7.0 \mathrm{~m} / \mathrm{s}$ & $5 \mathrm{M} \Omega$ & $\mathrm{Galloping}$ & $52.7 \mu \mathrm{W}$ \\
Song et al. [61] & Circular & Splitter plate & $7.0 \mathrm{~m} / \mathrm{s}$ & $5 \mathrm{M} \Omega$ & $\mathrm{Galloping}$ & $30.3 \mu \mathrm{W}$ \\
Wang et al. [64] & Circular & Y-shaped & $2.1 \mathrm{~m} / \mathrm{s}$ & $2 \mathrm{M} \Omega$ & $\mathrm{Galloping}$ & $1.2 \mathrm{~mW}$ \\
Ding et al. [67] & Circular & Fin-shaped & $6.9 \mathrm{~m} / \mathrm{s}$ & $0.2 \mathrm{M} \Omega$ & $\mathrm{VIV}$, galloping & $993.8 \mu \mathrm{W}$ \\
\hline
\end{tabular}

2.2. Cross-Section of Bluff Body. The FIV of a circular cylinder has been extensively studied both numerically $[72,73]$ and experimentally $[74,75]$. Cylinders with other common sections such as square, triangular, and D-shaped have also attracted attention. Ewere et al. [76] used a square cylinder as the piezoelectric energy harvester and proposed a model to predict the output power. The results coincided well with the experimental data. Zhang et al. [77] examined the harvested power of square cylinder at different attack angles. It was found that the attack angle of $45^{\circ}$ (diamond configuration) was the optimal one for energy harvesting. Wang et al. [78] investigated the performance of a galloping-based piezoelectric energy harvester with an isosceles triangular crosssection of different vertex angles. The obtuse angle of $130^{\circ}$ was found to be the most preferred vertex angle with relatively weak coupling. Zhu et al. [79] reported a numerical investigation into the flow around trapezoidal cylinders. Compared with a square cylinder, trapezoidal cylinders bear larger hydrodynamic forces, presenting a greater potential for energy harvesting. Sirohi and Mahadik [80] reported a maximum output power of $1.14 \mathrm{~mW}$ was achieved by a D-shaped cylinder at a wind velocity of $23.38 \mathrm{~m} / \mathrm{s}$.

Some tip bluff bodies with special sections are proposed to enhance the efficiency of energy harvesting, as shown in Figure 8. Kwon [81] designed a T-shaped piezoelectric cantilever and peak output power of $4 \mathrm{~mW}$ was obtained under a wind speed of $4 \mathrm{~m} / \mathrm{s}$. Liu et al. [82] proposed a threeblade bluff body with Y-type cross-section for energy harvesting, and the performance was significantly enhanced by adjusting the length of blades. Later, Liu et al. [83] designed a fork-shaped bluff body that has lower onset of wind speed and higher output power. Ewere et al. [84] proposed a bioinspired bluff body that possesses a sinusoidal wavy leading edge. It was observed that this kind of bluff body could harvest energy at an onset wind velocity $18 \%$ lower than square cylinder. Wang et al. [85] proposed a hybrid scavenger in the combination with circular and square crosssections, corresponding to different attack angles. The results showed such an energy scavenger at an appropriate attack angle could achieve a significant improvement in a wide 


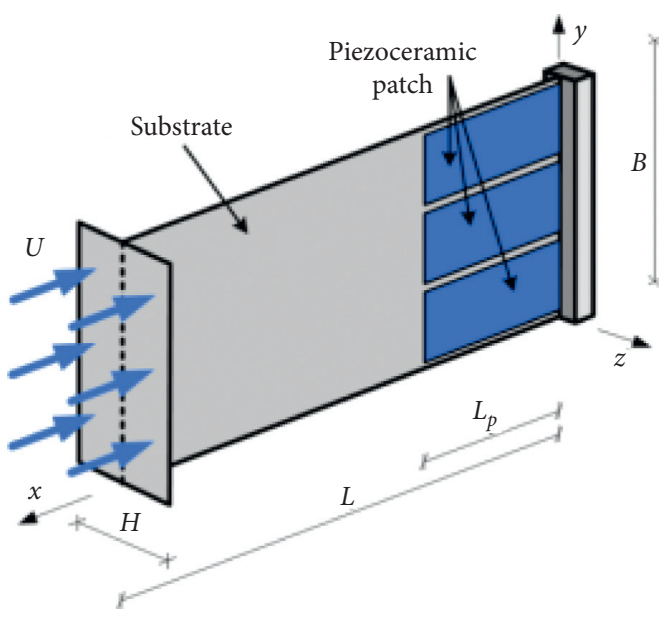

(a)

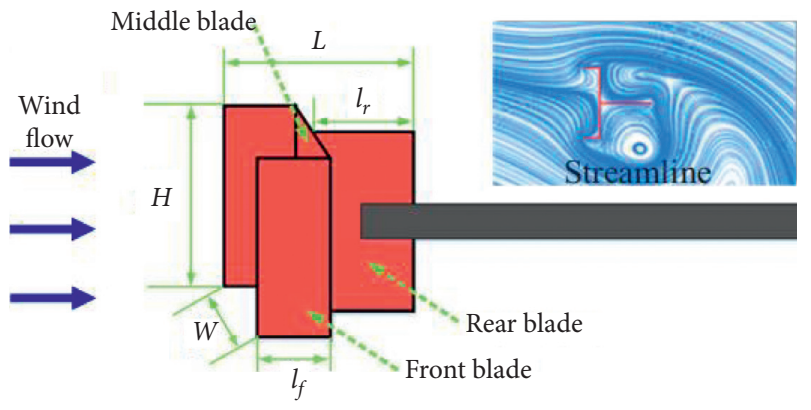

(c)

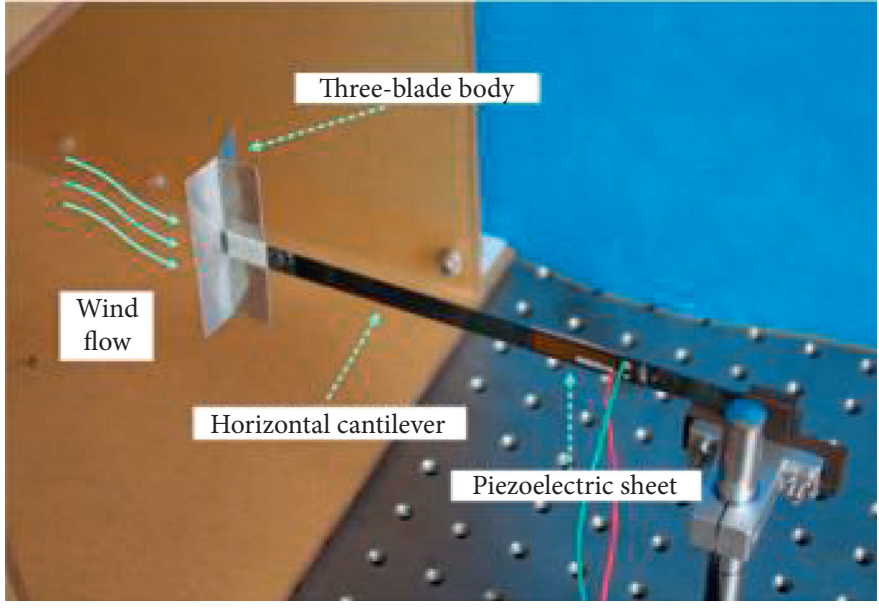

(b)
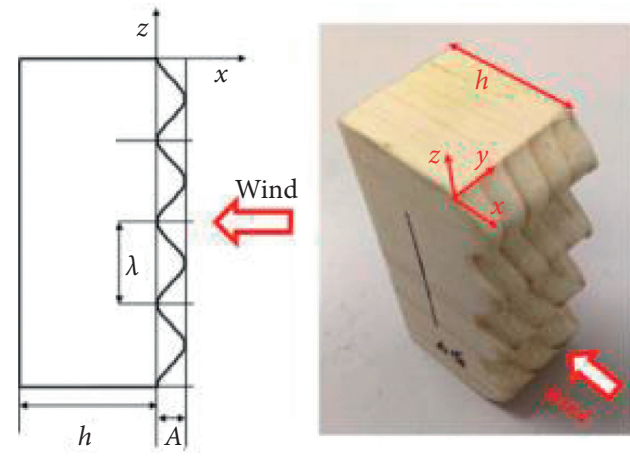

(d)

Figure 8: Bluff body with different cross-sections. (a) T-shape [81]. (b) Y-shape [82]. (c) Fork-shape [83]. (d) Bioinspired shape [84].

range of wind speeds, e.g., up to $71 \%$ augment in the maximum voltage output.

Some investigations were carried out to determine the optimal cross-section of bluff bodies. Abdelkefi et al. [86] developed a coupled nonlinear model to examine the effect of cross-sectional shapes including square, triangular, and D-shape on the performance of harvester. It was reported that the onset of galloping speed was sensitive to the crosssectional shape. The D-shaped cylinder achieved the highest harvested power at a constant resistance load of $10^{8} \Omega$. Kluger et al. [87] evaluated the behavior of square and trapezoidal cylinders as a transversely galloping-based wind energy harvester both experimentally and theoretically. The trapezoidal cylinder experienced about 5 times larger vibration displacement than that of the square cylinder. Through the wind-tunnel tests, Gkoumas et al. [88] observed the rectangular and cylindrical bluff bodies experienced the VIV response while the T-shaped bluff body subjected to the galloping phenomenon that extracted power up to $400 \mu \mathrm{W}$. Sun et al. [89] conducted similar experiments in a circulating water channel and found the maximum power density of $7.582 \mathrm{~mW} / \mathrm{cm}^{3}$ achieved through a D-shaped bluff body. Nevertheless, Yang et al. [90] reported the square cylinder reached the highest peak power of $8.4 \mathrm{~mW}$ and the onset wind speed could be as small as $2.5 \mathrm{~m} / \mathrm{s}$ in comparison with the rectangular, triangular, and D-shaped cylinders.

Table 4 compares the performance of harvesters in different shapes of cross-section in the previous studies. It is seen that the bluff body with sharp corners easily enters into the galloping branch, possibly attributed to the early separation of boundary layer and the existence of local recirculation region. As shown in Figure 9, taking the Y-shaped bluff body as an example, the vortices formed and shed periodically behind the two front blades, generating a pressure difference between the upper and lower surfaces of the rear blade and hence the periodically fluctuating transverse force [82]. Considering a rigid body undergoing both translational and rotational oscillations to its elastic center, the structural dynamic equations can be expressed as [91] 
TABLE 4: Summary of the effect of cross-section of bluff body on piezoelectric energy harvester.

\begin{tabular}{|c|c|c|c|c|c|c|c|}
\hline Researcher & Cross-section & Method & Flow velocity & Flow & Resistance load & Vibration type & Output power \\
\hline Dai et al. [72] & Circular & Theor. & $1.19 \mathrm{~m} / \mathrm{s}$ & Wind & $2.46 \mathrm{M} \Omega$ & VIV & $0.1 \mathrm{~mW}$ \\
\hline Dai et al. [73] & Circular & Theor. & $1.8 \mathrm{~m} / \mathrm{s}$ & Wind & $10 \mathrm{M} \Omega$ & VIV & $0.7 \mathrm{~mW}$ \\
\hline Jia et al. [75] & Circular & Exp. & $2.0 \mathrm{~m} / \mathrm{s}$ & Wind & $0.3 \mathrm{M} \Omega$ & VIV & $770.1 \mu \mathrm{W}$ \\
\hline Sun et al. [89] & Circular & Exp. & $0.5 \mathrm{~m} / \mathrm{s}$ & Current & $1 \mathrm{M} \Omega$ & VIV & $1.2 \mathrm{~mW}$ \\
\hline Akaydin et al. [74] & Circular & Theor. and Exp. & $1.19 \mathrm{~m} / \mathrm{s}$ & Wind & $2.46 \mathrm{M} \Omega$ & VIV & $0.1 \mathrm{~mW}$ \\
\hline Abdelkefi et al. [86] & Square & Theor. & $15.0 \mathrm{~m} / \mathrm{s}$ & Wind & $0.1 \mathrm{M} \Omega$ & Galloping & $0.8 \mathrm{~W}$ \\
\hline Ewere et al. [76] & Square & Exp. & $8.0 \mathrm{~m} / \mathrm{s}$ & Wind & $0.1 \mathrm{M} \Omega$ & Galloping & $12.2 \mathrm{~mW}$ \\
\hline Yang et al. [90] & Square & Exp. & $8.0 \mathrm{~m} / \mathrm{s}$ & Wind & $0.1 \mathrm{M} \Omega$ & Galloping & $8.4 \mathrm{~mW}$ \\
\hline Abdelkefi et al. [86] & Triangular & Theor. & $15.0 \mathrm{~m} / \mathrm{s}$ & Wind & $0.1 \mathrm{M} \Omega$ & Galloping & $3.8 \mathrm{~W}$ \\
\hline Sun et al. [89] & Triangular & Exp. & $0.8 \mathrm{~m} / \mathrm{s}$ & Current & $5 \mathrm{M} \Omega$ & Galloping & $10.0 \mathrm{~mW}$ \\
\hline Wang et al. [78] & Triangular & Exp. & $7.0 \mathrm{~m} / \mathrm{s}$ & Wind & $0.04 \mathrm{M} \Omega$ & Galloping & $269.1 \mathrm{~mW}$ \\
\hline Abdelkefi et al. [86] & D-shaped & Theor. & $15.0 \mathrm{~m} / \mathrm{s}$ & Wind & $1 \mathrm{M} \Omega$ & Galloping & $4.9 \mathrm{~W}$ \\
\hline Sun et al. [89] & D-shaped & Exp. & $0.8 \mathrm{~m} / \mathrm{s}$ & Current & $5 \mathrm{M} \Omega$ & Galloping & $15.9 \mathrm{~mW}$ \\
\hline Sirohi and Mahadik [80] & D-shaped & Theor. \& Exp. & $4.7 \mathrm{~m} / \mathrm{s}$ & Wind & $0.7 \mathrm{M} \Omega$ & Galloping & $1.2 \mathrm{~mW}$ \\
\hline Kwon $[81]$ & T-shaped & Theor. and Exp. & $15.0 \mathrm{~m} / \mathrm{s}$ & Wind & $4 \mathrm{M} \Omega$ & Galloping & $3.9 \mathrm{~mW}$ \\
\hline Liu et al. [82] & $Y$-shaped & Exp. & $5.0 \mathrm{~m} / \mathrm{s}$ & Wind & $1 \mathrm{M} \Omega$ & Galloping & $1.6 \mathrm{~mW}$ \\
\hline Liu et al. [83] & Fork-shaped & Exp. & $5.0 \mathrm{~m} / \mathrm{s}$ & Wind & $1 \mathrm{M} \Omega$ & Galloping & $1.2 \mathrm{~mW}$ \\
\hline Ewere et al. [84] & $\begin{array}{l}\text { Bioinspired- } \\
\text { shaped }\end{array}$ & Exp. & $10.5 \mathrm{~m} / \mathrm{s}$ & Wind & $0.5 \mathrm{M} \Omega$ & Galloping & $1.8 \mathrm{~mW}$ \\
\hline
\end{tabular}

${ }^{*}$ Exp. and Theor. denote the experimental and theoretical analysis, respectively.
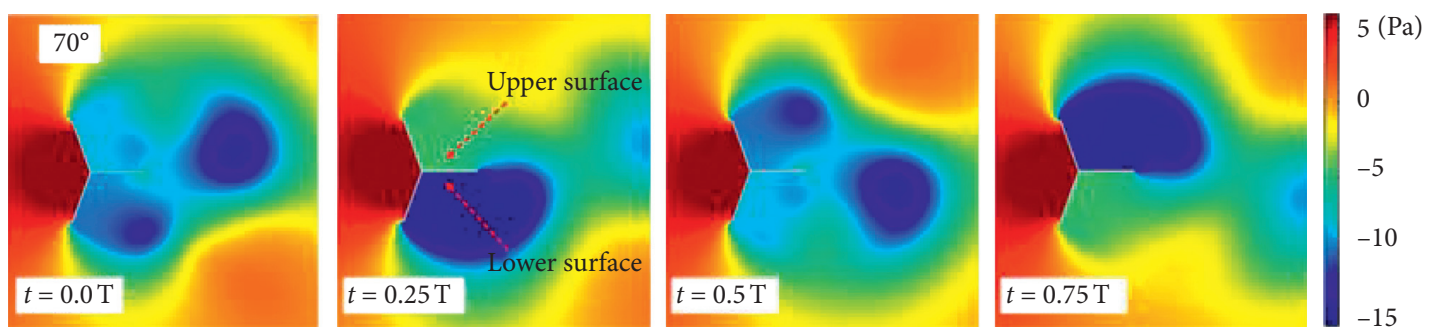

(a)
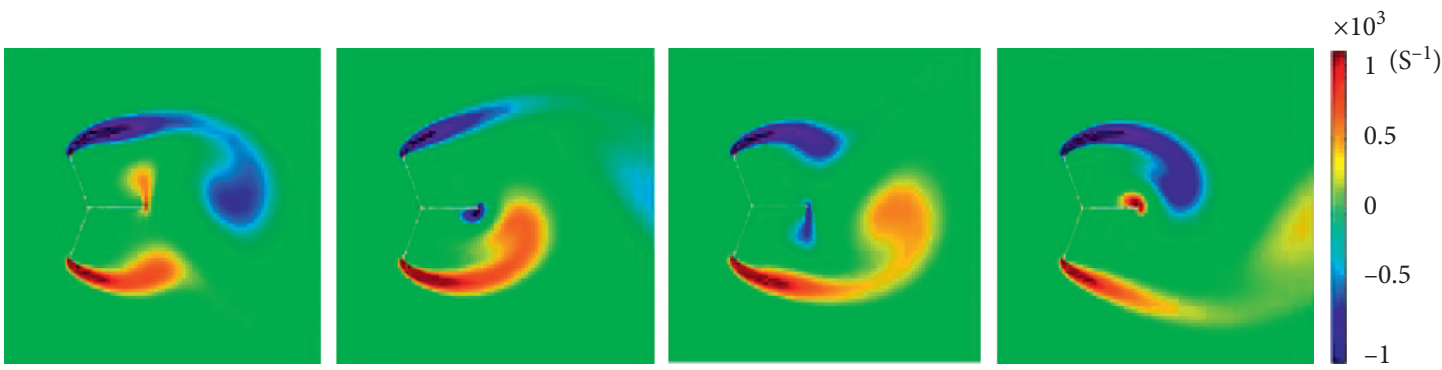

(b)

Figure 9: (a) Pressure distribution. (b) Vorticity contours of $Y$-shape bluff body [82].

$$
\left\{\ddot{d}+4 \pi \frac{\xi_{1}}{U_{r 1}} \dot{d}+4 \pi^{2} \frac{1}{U_{r 1}^{2}} \mathbf{d}=\frac{C_{D}}{2 m_{1}^{*}}, \ddot{d}+4 \pi \frac{\xi_{2}}{U_{r 2}} \dot{d}+4 \pi^{2} \frac{1}{U_{r 2}^{2}} \mathbf{d}=\frac{C_{L}}{2 m_{2}^{*}}, \ddot{d}+4 \pi \frac{\xi_{\theta}}{U_{r \theta}} \dot{d}+4 \pi^{2} \frac{1}{U_{r \theta}^{2}} \mathbf{d}=\frac{C_{M}}{2 I_{\theta}^{*}},\right.
$$

where $\mathbf{d}=[X, Y, \theta]$ is the displacement vector, $\xi_{i}(i=1,2)$ and $\xi_{\theta}$ are the translational and rotational damping ratios, $U_{r i}$ and $U_{r \theta}$ are the associated reduced velocities, $m_{i}^{*}$ is the mass ratio, and $I_{\theta}^{*}$ is the moment of inertia. $C_{D}, C_{L}$, and $C_{M}$ are the drag, lift, and moment coefficients, respectively. According to $\mathrm{Lu}$ et al. [92], the rotation angle and fluid moment are approximately predicted using harmonic functions:

$$
\left\{\begin{array}{l}
\theta(t)=\theta^{A} \sin (\omega t) \\
\frac{C_{M}(t)}{2 I_{\theta}^{*}}=M^{A} \sin (\omega t+\varphi)
\end{array}\right.
$$

where $\theta^{A}$ and $M^{A}$ denote the amplitude of stable rotation angle and fluid force moment, respectively; $\omega$ is the angular 
velocity and $\varphi$ is the phase difference between the fluid force moment and the rotation angle. Thus, the rotational equation is written as

$$
\frac{\mathrm{d}^{2} \theta(t)}{\mathrm{d} t^{2}}+\left(\frac{4 \pi \xi_{\theta}}{U_{r \theta}}-\frac{M^{A} \sin \varphi}{\omega \theta^{A}}\right) \frac{\mathrm{d} \theta(t)}{\mathrm{d} t}+\left(\frac{4 \pi^{2}}{U_{r \theta}^{2}}-\frac{M^{A} \cos \varphi}{\theta^{A}}\right) \theta(t)=0 .
$$

When the phase difference $\varphi$ is zero, the fluid force moment works as a negative stiffness, consequently leading to the large amplitude of rotational oscillation. By contrast, the appearance of phase difference $\pi$ indicates a positive stiffness. The antiphase between the rotation and the moment accounts for the decrease in the rotational amplitude.

Wang et al. [91] studied the VIV response of an elliptic cylinder in both transverse and azimuthal directions at different aspect ratios. It is reported that the lift and moment at lower $U_{r}$ are in-phase with the transverse and rotational motions, resulting in the increases of $Y$ and $\theta$, while the fluid forces become out of phase with the cylinder motions at higher $U_{r}$, leading to the decreases of $Y$ and $\theta$. They also observed that the transverse vibration frequency and the rotation frequency are identical to each other because the rotational effect is not strong enough to affect the vibrational response. In the reports by Zhu et al. [93, 94], the rotation is sufficiently strong for the Bach-type turbines and impeller. With the introduction of rotation, asymmetric flow wake is generated with faster downstream migration of vortices. As a result, the lock-in region of transverse oscillation is shortened or even disappeared under the rotational impact. Additionally, the angular position constantly varies over time during the coupling response, altering the projected area with respect to the oncoming flow and hence shifting the response frequency.

\section{Enhancement of Energy Harvesting}

3.1. Vortex Shedding-Induced Vibration. Vortex shedding usually occurs when flow pasts a bluff body. Researchers mainly focus on the vibration response of bluff body while paying less attention to the energy contained in the flow wake over past decades [95-98]. Some investigations have been conducted to examine the energy extraction from the vortex shedding-induced vibration (VSIV) via piezoelectric harvesters. Usman et al. [99] placed a stationary cylinder in front of a circular cylinder-based wind energy harvester. An optimal spacing was identified as 3 times the diameter of the circular cylinder. Alhadidi and Daqaq [100] evaluated the behavior of a bistable piezoelectric cantilever harvester with a square bluff body that was immersed in the wake of a stationary rectangular rod. Their investigations indicated the bistability can be used to improve the steady-state bandwidth considerably. Wang et al. [101] used a stationary square column upstream of the energy harvester to generate wake shedding. Such configuration significantly broadened the bandwidth of the energy harvester as compared to a single bluff body. Rezaei and Talebitooti [102] proposed a PZT (piezoelectric ceramic transducer) energy harvester composed of an array of bimorphs placed downstream of a bluff body. The results confirmed that such a system had a wider lock-in region and a larger conversion factor, compared to the typical wake-galloping energy harvesters. $\mathrm{Hu}$ et al. [103] proposed a VSIV piezoelectric energy harvester, as shown in Figure 10. A bimorph piezoelectric vibrator was attached on the flapping sheet that was placed in the flow wake of a bluff body. The performance of this harvester was found to be dependent on the flow velocity and the diameter of bluff body. Weinstein et al. [104] investigated the interaction between a fin attached at the end of a piezoelectric cantilever and the vortex shedding of a bluff body placed upstream of the former. In an air duct of diameter $15 \mathrm{~cm}$, the generated power was $200 \mu \mathrm{W}$ and $3 \mathrm{~mW}$ at flow speeds of $2.5 \mathrm{~m} / \mathrm{s}$ and $5 \mathrm{~m} / \mathrm{s}$, respectively.

Some studies focus on the relationship between the vortex shedding and the piezoelectric patch rather than the accompanying motion with the bluff body. Akaydm et al. [105] evaluated the performance of a generator with a shortlength piezoelectric beam placed in the wake of a circular cylinder. The results showed that the driving forces of the beam originated from two aspects: the flow impingement caused by the passing vortices on one side of the beam and the low-pressure core region of the vortices at the opposite side of the beam. Goushcha et al. [106] designed a custom experimental facility to study the interaction of individual and multiple vortices with the piezoelectric beam. They found that the strain exerted on the beam became larger when the vortex arrival rate matched the natural frequency of the beam. As shown in Figure 11, Shan et al. [107] tested a piezoelectric harvester immersed in the wake of a circular cylinder. Maximum output power of $1.32 \mu \mathrm{W}$ was achieved with a power density of $1.1 \mathrm{~mW} / \mathrm{m}^{2}$ when the water velocity was $0.5 \mathrm{~m} / \mathrm{s}$.

Compared to the single harvester, it has some superior advantages by utilizing shedding vortex to excite the vibration and hence to convert the vibration energy into electricity through the piezoelectric effect. Firstly, such configuration can considerably widen the bandwidth of the lock-in region or excite the wake-galloping response, resulting in the enhancement of energy harvesting. Secondly, the efficiency can be significantly increased by tuning the frequency of vortex shedding to match the resonant vibration frequency.

3.2. Interference of Multiple Harvesters. As the advantages are presented when the harvester is placed behind a bluff body, some researchers have been devoted to investigating the influence of wake interference [108, 109]. Zhu et al. [110] investigated the effect of center-to-center spacing ratio $(L / D)$ on the FIV of two rigidly coupled tandem cylinders of unequal diameters. It was reported that both the hydrodynamic forces and response amplitudes are augmented at $L / D=3.75$ and $L / D=6.0$ when $U_{r}>8$ compared to an isolated cylinder. Abdelkefi et al. [111] evaluated the output power of a square piezoaeroelastic energy harvester placed downstream of an oscillating circular cylinder. A wake-induced galloping was observed for the square cylinder with a widened operating range of wind speed. $\mathrm{Hu}$ et al. [112] 


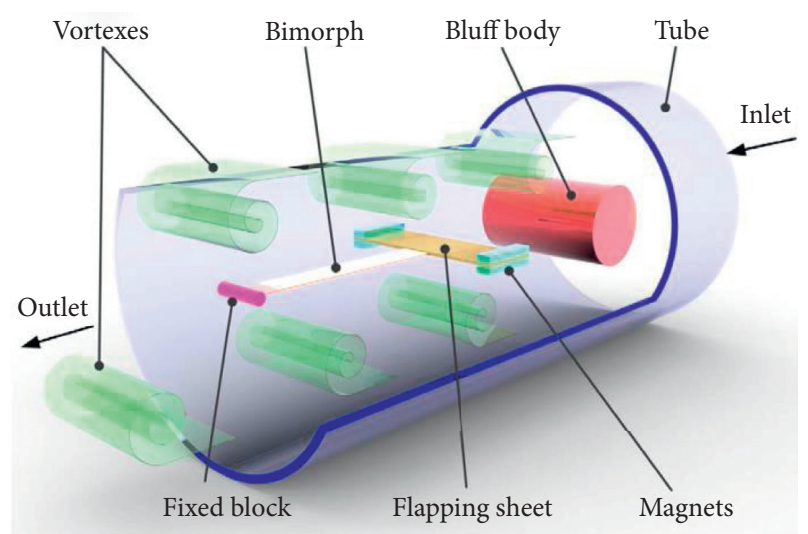

FIGURE 10: Schematic of the VSIV piezoelectric energy harvester [103].

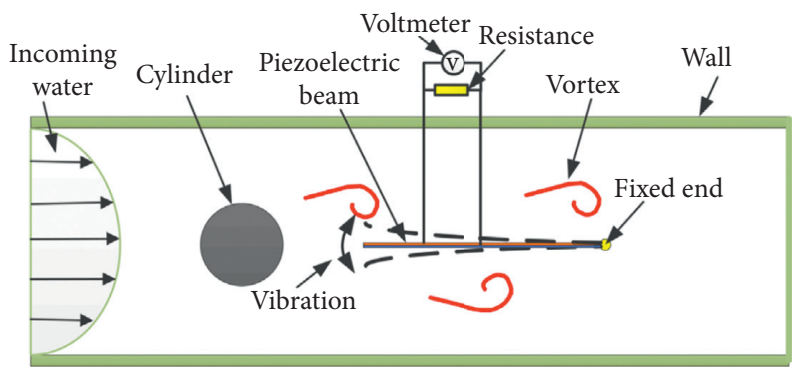

FIGURE 11: Schematic of the piezoelectric harvester downstream of a bluff body [107].

evaluated the performance of twin adjacent galloping-based piezoelectric harvesters based on mutual interference. The total output power of them in staggered configuration was up to 2.2 times larger than the sum of the two isolated ones. Shan et al. [113] investigated two underwater double piezoelectric harvesters arranged in series. When the spacing distance was $2 D$, the maximum output was increased by up to $99 \%$ in comparison with a single one. Similarly, Zhou and Wang [114] tested a dual serial energy harvester consisted of two identical piezoelectric cantilevers. The experimental results indicated that the effective wind speed range and the output power of the coupled harvester were as many as 2.67 times and 6.79 times of the associated values of a single harvester, respectively. Inspired by tree trunks swaying in wind, Hobbs and $\mathrm{Hu}$ [115] proposed a tree-like energy harvester and arranged four harvesters in tandem, as shown in Figure 12. The maximum power was attained with the center-to-center spacing of $3.3 \mathrm{D}$ under a specific flow speed when the vortex shedding frequency was 1.6 times the natural frequency of the cylinders.

Unlike the wake interference, Zhang et al. [116] examined the performance of an oscillating circular cylinder placed upstream of an interference circular cylinder. As compared to the isolated cylinder, the harvested power was greatly enhanced with a widened bandwidth of the resonance region. They further investigated the effect of the shape of the downstream cylinder [117], including square, circular, triangular, and D-shaped, as displayed in Figure 13.

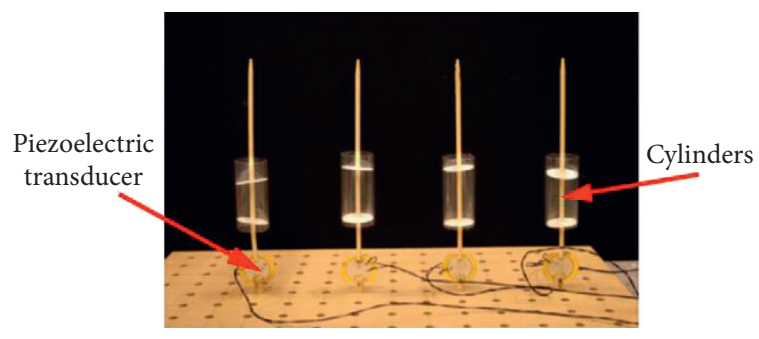

FIGURE 12: Photograph of four piezoelectric devices in tandem [115].

In the presence of a square interference cylinder with a spacing ratio of 0.9 , the average power of $803.4 \mathrm{~mW}$ was attained at a wind speed of $2.36 \mathrm{~m} / \mathrm{s}$ and the synchronization region was broadened 2.8 times larger than that of a single cylinder.

3.3. Cantilever Beam Structure. The geometry and stiffness of the cantilever beam also affect the output performance. Sun et al. [118] proposed a vibro-wind galloping energy harvester with a nested configuration, as shown in Figure 14. They concluded that the inner bluff body played an important role in enhancing the output power, particularly under relatively high wind speed. A distinct increase of $27.8 \%$ in the power density was achieved. Zhao et al. [119] replaced the inner bluff body with a magnet in the opposite position while another magnet was attached on the outer bluff body. The translational galloping occurred, and the stiffness nonlinearity was created by the magnetic interaction. This device required a lower cut-in speed of $1 \mathrm{~m} / \mathrm{s}$ but output higher power. Song et al. [120] designed a serial energy harvesting system (Figure 15) that was fixed upstream. This system consisted of two piezoelectric beams and two cylinders arranged in tandem, and the downstream piezoelectric beam was connected to the upstream cylinder. The maximum output power of $21.86 \mu \mathrm{W}$ was obtained under a water flow velocity of $0.31 \mathrm{~m} / \mathrm{s}$. Zhao and Wang [49] attached a beam stiffener to the substrate of harvester that worked as an electromechanical coupling magnifier, as shown in Figure 16. Several times increase of power and almost double extraction efficiency were achieved. Wang et al. [121] proposed a cross-coupled dual-beam structure for multidirectional energy harvesting, as depicted in Figure 17. The upper and bottom beams could generate a maximum output power of $6.77 \mu \mathrm{W}$ and $56.65 \mu \mathrm{W}$, respectively, in case B.

3.4. Stable System by Magnetic Force. Some researchers introduced magnet-induced nonlinearity into the piezoelectric energy harvester. According to the pairs of magnets, bistable and tristable energy harvesting systems are proposed. Ferrari et al. [21] and Vocca et al. [122] experimentally measured both the beam deflection and the output voltage under the excitation of random vibrations with various degrees of nonlinearity. The output voltage at a parity of mechanical excitation was improved remarkably when the system was bistable. Zhang et al. [123] proposed a bistable piezoelectric harvester by varying the relative position of two magnets, as 


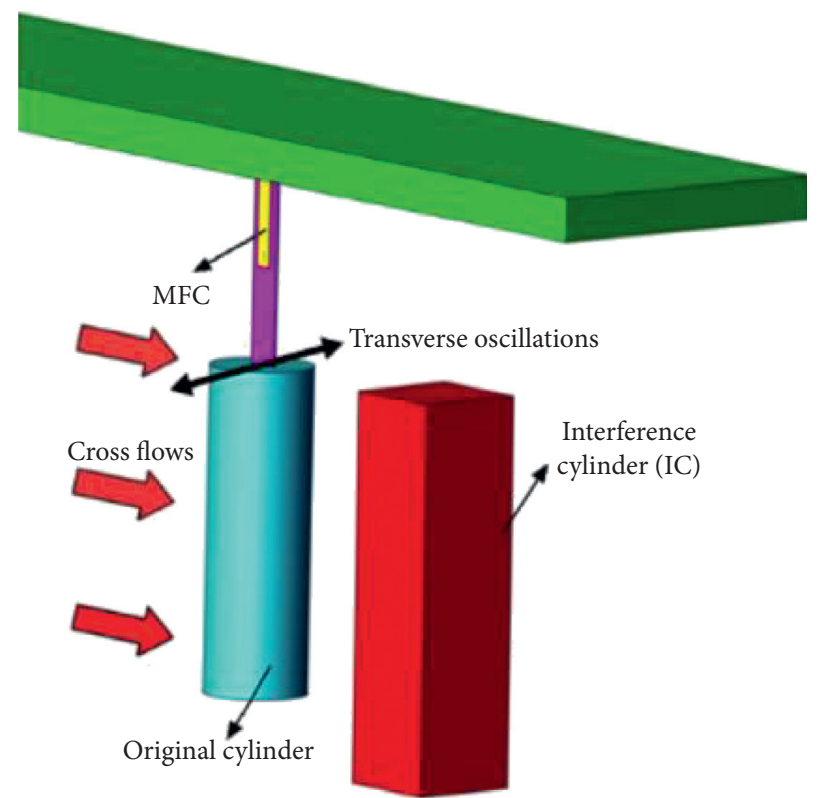

(a)

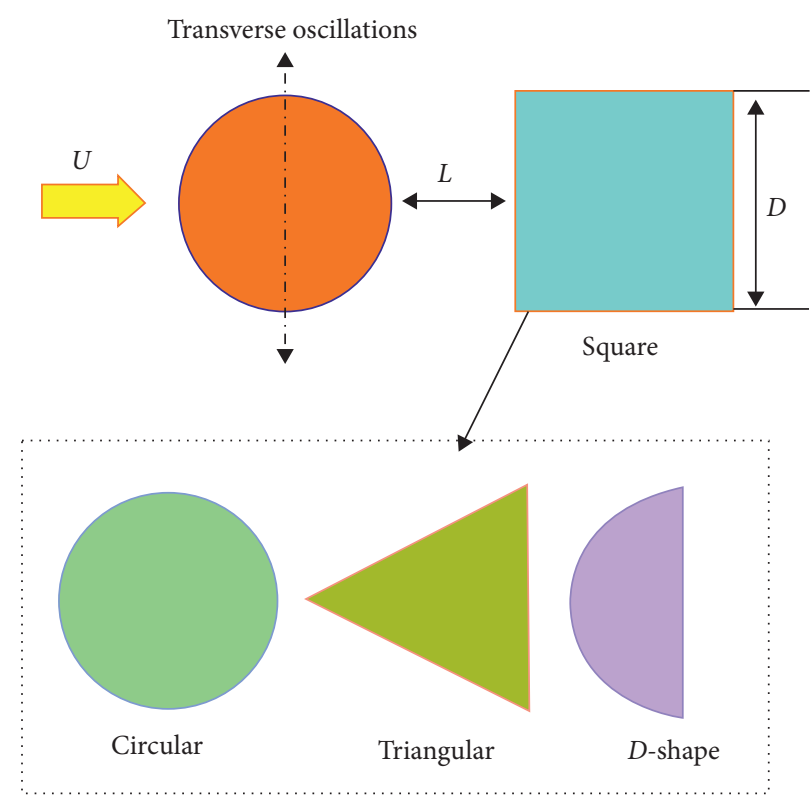

(b)

FIgUre 13: (a) VIV energy harvester. (b) The downstream cylinder in different shapes [117].

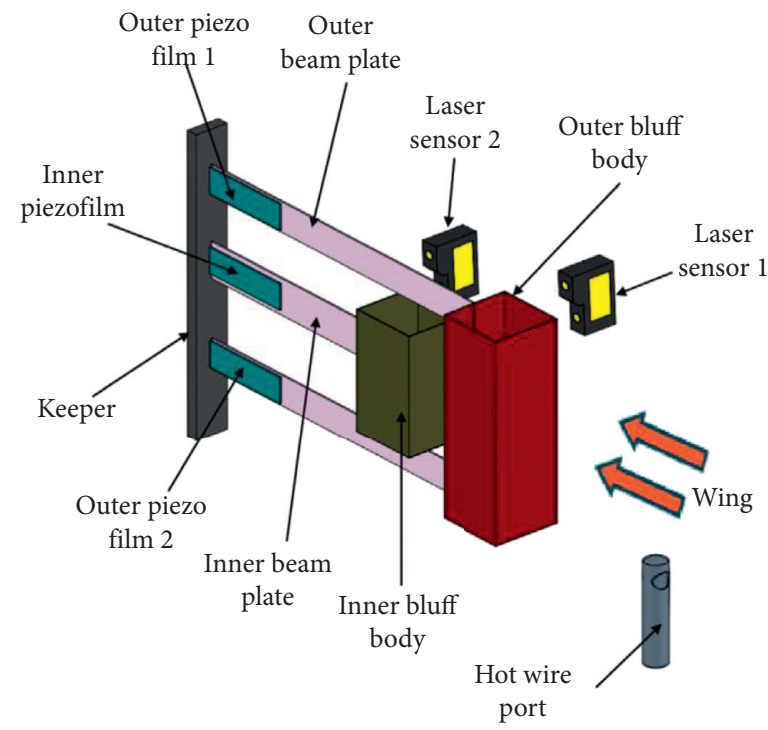

FIGURE 14: The energy harvester with a nested configuration [118].

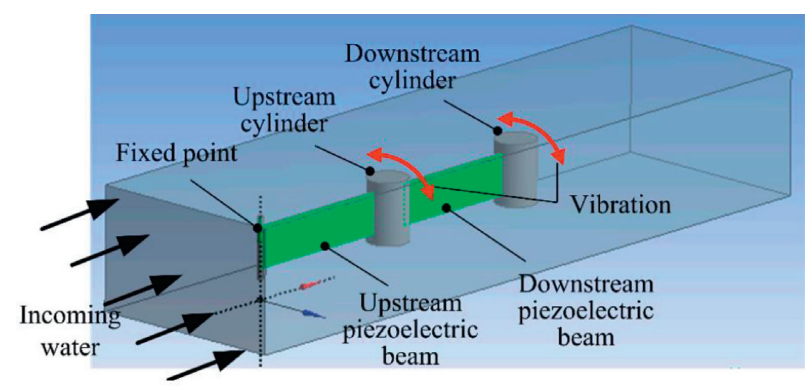

FIgURE 15: The energy harvester with two serial cantilever beams and cylinders [120]. 


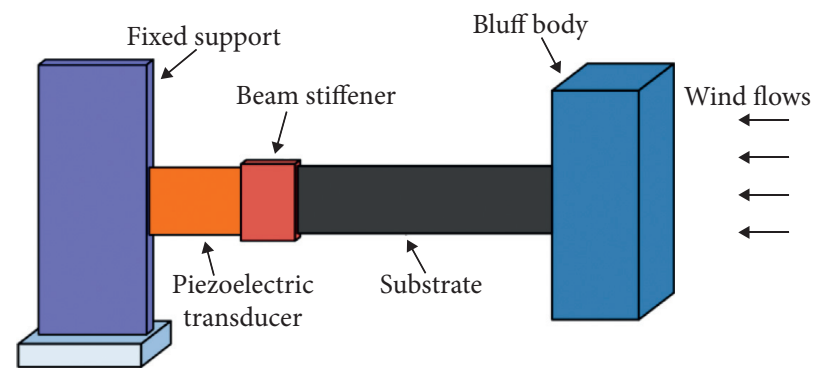

FIGURE 16: The prototype of energy harvester attached with a beam stiffener [49].

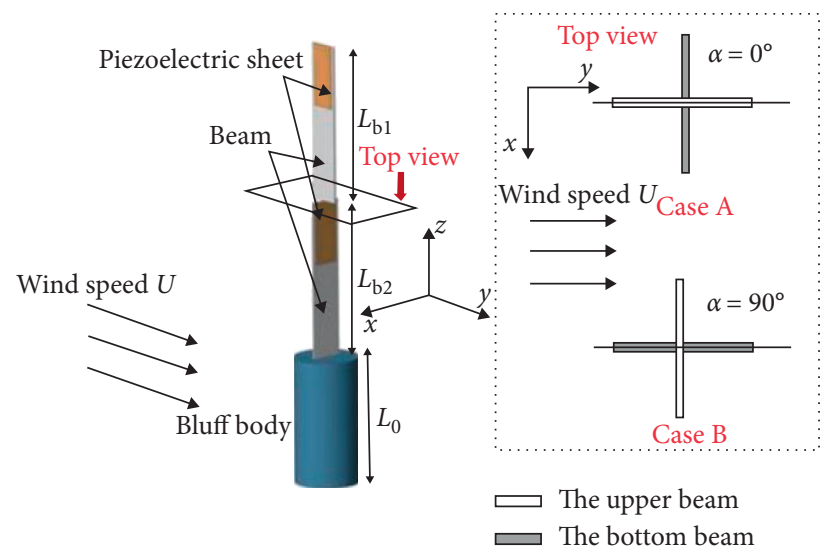

Figure 17: The cross-coupled dual-beam structure [121].

shown in Figure 18. This harvester significantly broadened the synchronization region and increased the harvested power by $138 \%$ and $29 \%$, respectively. Yang et al. [47] coupled two identical harvesters and each of them supported a prism bluff body embedded with a magnet at its tip, as plotted in Figure 19. It was reported that the onset wind speed of galloping was reduced up to $41.9 \%$. Wang et al. [124] conducted similar investigations and intrawell, chaotic, and interwell oscillations were observed with respect to low, medium, and high wind speed intervals, respectively. As shown in Figure 20, compared to the bistable energy harvester, the tristable energy harvester has three stable equilibrium positions $(A, E$, and $C$ ) and two unstable equilibrium positions ( $B$ and $D$ ), leading to shallower potential wells than that of bistable energy harvester as the potential energy is distributed into three potential wells $[125,126]$. Due to the effect of magnetic force, the restoring force becomes more nonlinear, and the electromechanical dynamic modeling could be expressed as

$$
\left\{\begin{array}{l}
M \stackrel{\dot{\mathrm{t}}}{x}(t)+C \dot{x}(t)+F_{r}-\theta V(t)=F(t), \\
C_{p} \dot{V}(t)+\frac{V(t)}{R}+\theta \dot{x}(t)=0,
\end{array}\right.
$$

where $M$ is the equivalent mass, $C$ is the equivalent damping, $F_{r}$ is the equivalent nonlinear restoring force [125], $F(t)$ is the external mechanical force as the excitation, $\theta$ is the equivalent electromechanical coupling coefficient, $C_{p}$ is the equivalent capacitance of the piezoelectric materials, $R$ is the

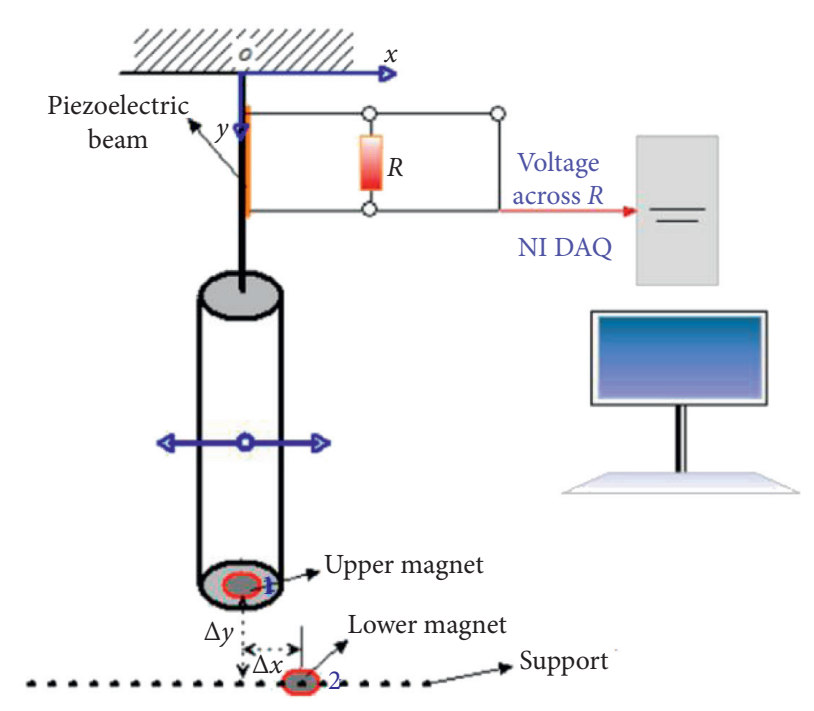

FIgURE 18: The VIV-based bistable energy harvesting system [123].

load resistance, $x(t)$ is the tip displacement of the harvester in the transverse direction, and $V(t)$ is the voltage across the electrical load. Wherein, the restoring force $F_{r}$ could be defined as a polynomial of the horizontal displacement:

$$
F_{r}=n_{0}+n_{1} x+n_{2} x^{2}+\cdots+n_{n} x^{n}
$$

where $n_{0}, n_{1}, n_{2}, \ldots, n_{n}$ are polynomial coefficients and $x$ is the tip displacement of the harvester. After comparing the stiffness of linear and nonlinear energy harvesters, Zhou 

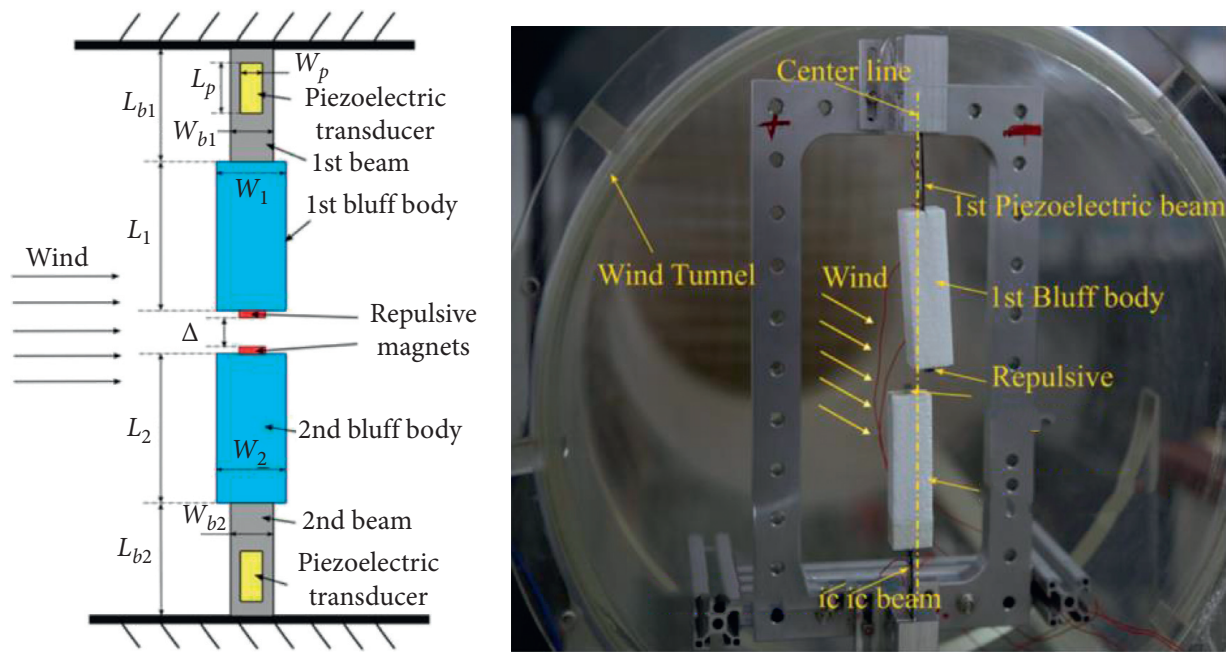

Figure 19: The double-beam piezo-magneto-elastic energy harvester [47].

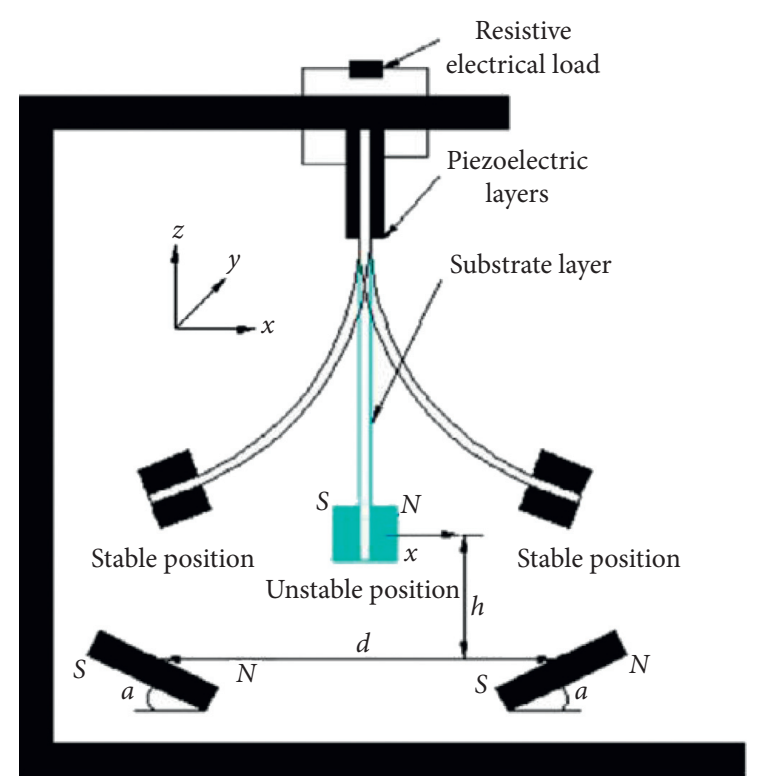

(a)

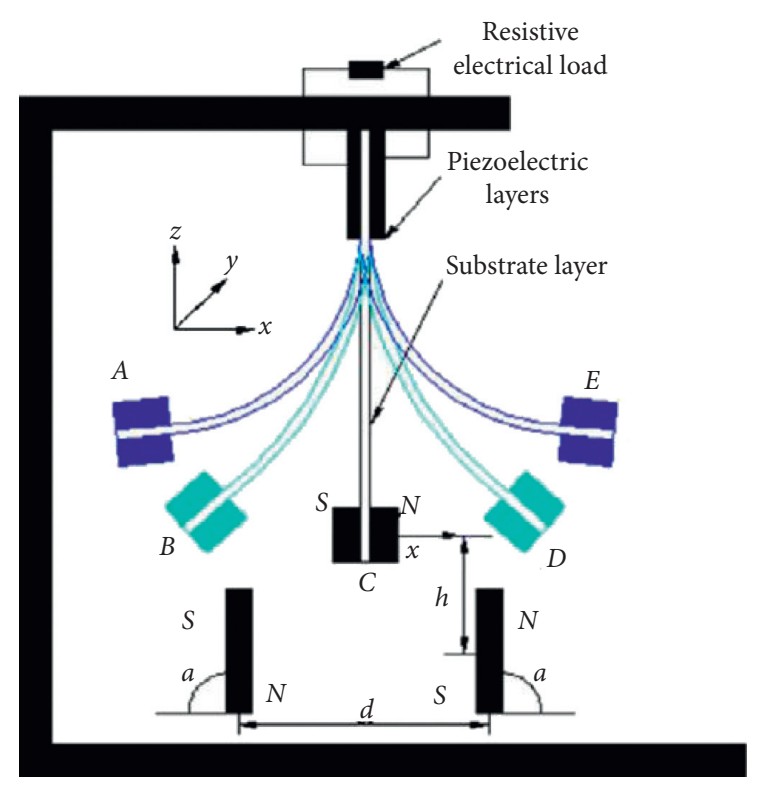

(b)

Figure 20: Nonlinear energy harvesting system. (a) Bistable. (b) Tristable [125].

et al. [125] reported that a negative stiffness value of the nonlinear harvester indicates the harvester locates in the unstable range and spontaneously moves to stable equilibrium position.
Table 5 summarizes the commonly used piezoelectric materials and the associated devices utilizing the flow-induced vibration. The inorganic and composite materials are mainly adopted for energy harvesting. Among them, the 
TABLE 5: Summary of piezoelectric materials and associated devices reported in the previous literature.

\begin{tabular}{|c|c|c|c|c|c|c|}
\hline $\begin{array}{l}\text { Piezoelectric } \\
\text { materials }\end{array}$ & Investigators & Materials & $\begin{array}{l}\text { Capacitance } \\
\quad(\mathrm{nF})\end{array}$ & $\begin{array}{l}\text { Size }(\text { length } \times \text { width } \times \text { thickness, } \\
\quad \mathrm{mm} \times \mathrm{mm} \times \mathrm{mm})\end{array}$ & Configuration & $\begin{array}{c}\text { Maximum } \\
\text { output } \\
\text { performance }\end{array}$ \\
\hline \multirow{4}{*}{ Inorganic } & $\begin{array}{l}\text { Yang et al. } \\
\text { [47] }\end{array}$ & PZT-5 & 26 & $30 \times 10 \times 0.4$ & $\begin{array}{l}\text { Cantilever with } \\
\text { magnetic mass }\end{array}$ & $\begin{array}{l}2.8 \mathrm{~V} \text { at } 3.7 \mathrm{~m} / \mathrm{s} \\
\text { (wind) }\end{array}$ \\
\hline & $\begin{array}{l}\text { Zhao et al. } \\
\text { [119] }\end{array}$ & $\begin{array}{l}\text { DuraAct P- } \\
\text { 876.A12 }\end{array}$ & 90 & $61 \times 30 \times 0.5$ & $\begin{array}{l}\text { Cantilever with tip } \\
\text { mass (bimorph } \\
\text { beam) }\end{array}$ & $\begin{array}{l}8.4 \mathrm{~mW} \text { at } 8 \mathrm{~m} / \mathrm{s} \\
\text { (wind) }\end{array}$ \\
\hline & Kwon [81] & $\begin{array}{l}\text { PZT-5A- } \\
\text { M-2814-P2 }\end{array}$ & 25.7 & $28 \times 14 \times \mathrm{N} / \mathrm{A}$ & $\begin{array}{l}\text { Cantilever with tip } \\
\text { mass (unimorph } \\
\text { beam) }\end{array}$ & $\begin{array}{c}3.9 \mathrm{~mW} \text { at } 15 \mathrm{~m} / \\
\mathrm{s} \text { (wind) }\end{array}$ \\
\hline & $\begin{array}{l}\text { Yang et al. } \\
{[90]}\end{array}$ & $\begin{array}{l}\text { DuraAct P- } \\
\text { 876.A12 }\end{array}$ & 90 & $61 \times 30 \times 0.5$ & $\begin{array}{l}\text { Cantilever with } \\
\text { magnetic mass }\end{array}$ & $\begin{array}{c}6.3 \mathrm{~mW} \text { at } 5 \mathrm{~m} / \mathrm{s} \\
\text { (wind) }\end{array}$ \\
\hline \multirow{9}{*}{ Composite } & Hu et al. [57] & $\begin{array}{l}\text { MFC- } \\
\text { M8514-P2 }\end{array}$ & N/A & N/A & $\begin{array}{c}\text { Cantilever with tip } \\
\text { mass (unimorph } \\
\text { beam) }\end{array}$ & $\begin{array}{c}71.4 \mu \mathrm{W} \text { at } 5.9 \mathrm{~m} / \\
\mathrm{s} \text { (wind) }\end{array}$ \\
\hline & $\begin{array}{l}\text { Wang et al. } \\
\text { [64] }\end{array}$ & $\begin{array}{l}\text { MFC-2807- } \\
\quad \text { P2 }\end{array}$ & 15.7 & $37 \times 11 \times 0.3$ & $\begin{array}{l}\text { Cantilever with tip } \\
\text { mass (unimorph } \\
\text { beam) }\end{array}$ & $\begin{array}{c}1.2 \mathrm{~mW} \text { at } 2.1 \mathrm{~m} / \\
\mathrm{s} \text { (wind) }\end{array}$ \\
\hline & Jia et al. [75] & $\begin{array}{l}\text { MFC-M- } \\
8507-\mathrm{P} 2\end{array}$ & 23.556 & $85 \times 7 \times 0.3$ & $\begin{array}{c}\text { Cantilever with tip } \\
\text { mass (unimorph } \\
\text { beam) }\end{array}$ & $\begin{array}{c}770.1 \mu \mathrm{W} \text { at } \\
2.0 \mathrm{~m} / \mathrm{s} \text { (wind) }\end{array}$ \\
\hline & Sun et al. [89] & $\begin{array}{l}\text { MFC-M- } \\
5628-\mathrm{P} 2\end{array}$ & 14.04 & $\mathrm{~N} / \mathrm{A} \times 28 \times 0.3$ & $\begin{array}{l}\text { Cantilever with tip } \\
\text { mass (unimorph } \\
\text { beam) }\end{array}$ & $\begin{array}{c}15.9 \mathrm{~mW} \text { at } \\
0.8 \mathrm{~m} / \mathrm{s} \text { (current) }\end{array}$ \\
\hline & $\begin{array}{l}\text { Ewere et al. } \\
{[76]}\end{array}$ & $\begin{array}{l}\text { MFC- } \\
\text { M0714-P2 }\end{array}$ & 422 & $85 \times 28 \times 0.3$ & $\begin{array}{l}\text { Cantilever with tip } \\
\text { mass (unimorph } \\
\text { beam) }\end{array}$ & $\begin{array}{c}12.2 \mathrm{~mW} \text { at } 8 \mathrm{~m} / \\
\mathrm{s} \text { (wind) }\end{array}$ \\
\hline & $\begin{array}{l}\text { Shan et al. } \\
\text { [107] }\end{array}$ & MFC & 89.5 & $85 \times 14 \times 0.3$ & $\begin{array}{l}\text { Pure unimorph } \\
\text { beam }\end{array}$ & $\begin{array}{c}1.32 \mu \mathrm{W} \text { at } \\
0.5 \mathrm{~m} / \mathrm{s} \text { (current) }\end{array}$ \\
\hline & $\begin{array}{l}\text { Zhang et al. } \\
\text { [116] }\end{array}$ & $\begin{array}{l}\text { MFC- } \\
\text { M2807-P2 }\end{array}$ & N/A & $62 \times 20 \times 0.6$ & $\begin{array}{c}\text { Cantilever with tip } \\
\text { mass (unimorph } \\
\text { beam) }\end{array}$ & $\begin{array}{l}1.6 \mathrm{~mW} \text { at } 5 \mathrm{~m} / \mathrm{s} \\
\text { (wind) }\end{array}$ \\
\hline & $\begin{array}{l}\text { Shan et al. } \\
\text { [113] }\end{array}$ & $\begin{array}{c}\text { MFC-8514- } \\
\text { P2 }\end{array}$ & N/A & $80 \times 18 \times 0.3$ & $\begin{array}{l}\text { Cantilever with tip } \\
\text { mass (unimorph } \\
\text { beam) }\end{array}$ & $\begin{array}{c}27.65 \mathrm{~V} \text { at } \\
0.58 \mathrm{~m} / \mathrm{s} \\
\text { (current) }\end{array}$ \\
\hline & $\begin{array}{c}\text { Zhang et al. } \\
\text { [123] }\end{array}$ & $\begin{array}{c}\text { MFC- } \\
\text { M2807-P2 }\end{array}$ & N/A & $60 \times 10 \times 0.6$ & $\begin{array}{l}\text { Cantilever with } \\
\text { magnetic mass }\end{array}$ & $\begin{array}{c}142 \mu \mathrm{W} \text { at } 3.2 \mathrm{~m} / \\
\mathrm{s} \text { (wind) }\end{array}$ \\
\hline
\end{tabular}

N/A denotes unavailable.

microfiber composite (MFC) material is favored due to its good bending performance and considerable piezoelectric capacity.

\section{Conclusions and Outlooks}

This paper briefly reviews the associated technologies of piezoelectric energy harvesting from flow-induced vibration. The piezoelectric material, configuration, and size as well as ambient flow have significant effects on the performance of harvesters. To enhance the structural vibration response and hence improve the energy harvesting efficiency, a variety of measures have been proposed and investigated in the past decades, including the irregularity and alteration of the cross-section of bluff bodies, utilization of wake flow and interference, modification and rearrangement of cantilever beams, and introduction of magnetic force. The combination of two or more of them is proved to be superior to a single scheme. From the reported studies, the energy harvesting from flow-induced vibrations presents a remarkable application potential, providing a feasible solution for the increasing demand for renewable energy. Nevertheless, there are still some open questions to be revealed as follows:

(1) The majority of present piezoelectric energy harvesting experiments were conducted in wind tunnel rather than water channel, as sealing and packing do not need to be considered in the wind tunnel. Nevertheless, marine current and wave energy significantly exceed the wind energy and possess a relatively stable flow direction and velocity. Thus, extensive studies are required to evaluate the piezoelectric energy harvesters in water currents and uncover the underlying physics of fluid-structure interaction.

(2) Although the energy harvesting is improved by the aforementioned measures, understanding of the underlying mechanisms of wake interference of multiple structures, the interaction between vortices and flexible piezoelectric layer, and coupling of the 
magnetic field and flow field, to name a few, are still limited. Besides, the flow direction is unchangeable with a fixed value in the reported literature, deviating from the actual environment.

(3) Nonlinear technology is used to improve the efficiency of energy harvesting. Nonetheless, an accurate description of nonlinear forces is required to quantify its contribution.

Compared to the conventional turbine generators, the output power of piezoelectric energy harvester is much less although the cut-in velocity lower than the former. Thus, it is more suitable for small-scale power generation. The optimization of piezoelectric materials and the arrangement of piezoelectric generators are still worth studying to broaden its application.

\section{Conflicts of Interest}

The authors declare that they have no conflicts of interest.

\section{Acknowledgments}

The research work was supported by the National Natural Science Foundation of China (no. 51979238), the Open Research Fund of State Key Laboratory of Coastal and Offshore Engineering, Dalian University of Technology (no. LP1930), and the PetroChina Southwest Oil and Gasfield Company Postdoctoral Program (no. 20200302-07). The authors appreciate the support from the Deep-Sea Cloud Computing Center of China and the Laboratory of Offshore Oil and Gas Engineering at Southwest Petroleum University.

\section{References}

[1] J. A. Paradiso and T. Starner, "Energy scavenging for mobile and wireless electronics," IEEE Pervasive Computing, vol. 4, no. 1, pp. 18-27, 2005.

[2] C. R. Lawrence, F. Louis, M. G. Evan et al., "Generating electricity while walking with loads," Science, vol. 309, no. 5741, pp. 1725-1728, 2005.

[3] L.-C. Zhao, H.-X. Zou, G. Yan et al., "A water-proof magnetically coupled piezoelectric-electromagnetic hybrid wind energy harvester," Applied Energy, vol. 239, pp. 735-746, 2019.

[4] D. L. Nicholas and P. E. Brenden, "Hydrokinetic energy conversion: technology, research, and outlook," Renewable and Sustainable Energy Reviews, vol. 57, pp. 1245-1259, 2016.

[5] S. P. Beeby, M. J. Tudor, and N. M. White, "Energy harvesting vibration sources for microsystems applications," Measurement Science and Technology, vol. 17, pp. 175-195, 2006.

[6] V. P. Dragunov, D. J. Ostertak, K. G. Pelmenev et al., "Electrostatic vibrational energy converter with two variable capacitors," Sensors and Actuators A: Physical, vol. 318, Article ID 112501, 2021.

[7] D. Q. Tan, "Review of polymer-based nanodielectric exploration and film scale-up for advanced capacitors," Advanced Functional Materials, vol. 30, Article ID 1808567, 2019.

[8] J. Hu, S. F. Zhang, and B. T. Tang, "Rational design of nanomaterials for high energy density dielectric capacitors via electrospinning," Energy Storage Materials, vol. 37, pp. 530-555, 2021.

[9] D. A. Barkas, C. S. Psomopoulos, P. Papageorgas, K. Kalkanis, D. Piromalis, and A. Mouratidis, "Sustainable energy harvesting through triboelectric nano-generators: a review of current status and applications," Energy Procedia, vol. 157, pp. 999-1010, 2019.

[10] N. Sezer and M. Koç, "A comprehensive review on the stateof-the-art of piezoelectric energy harvesting," Nano Energy, vol. 80, Article ID 105567, 2021.

[11] Z. Yang, S. Zhou, J. Zu, and D. Inman, "High-performance piezoelectric energy harvesters and their applications," Joule, vol. 2, no. 4, pp. 642-697, 2018.

[12] R. A. Steven and A. S. Henry, "A review of power harvesting using piezoelectric materials (2003-2006)," Smart Materials and Structures, vol. 16, no. 3, 2007.

[13] H. Kawai, "The piezoelectricity of poly (vinylidene fluoride)," Japanese Journal of Applied Physics, vol. 8, no. 7, pp. 975-976, 1969.

[14] S. B. Lang and S. Muensit, "Review of some lesser-known applications of piezoelectric and pyroelectric polymers," Applied Physics A, vol. 85, no. 2, pp. 125-134, 2006.

[15] J. Hao, W. Li, J. Zhai, and H. Chen, "Progress in high-strain perovskite piezoelectric ceramics," Materials Science and Engineering: Reports, vol. 135, pp. 1-57, 2019.

[16] B. Jaffe, R. S. Roth, and S. Marzullo, "Piezoelectric properties of lead zirconate-lead titanate solid-solution ceramics," Journal of Applied Physics, vol. 25, no. 6, pp. 809-810, 1954.

[17] T. J. Johnson, D. Charnegie, W. W. Clark et al., "Energy harvesting from mechanical vibrations using piezoelectric cantilever beams," Smart Structures and Materials: Damping and Isolation, vol. 6169, Article ID 61690D-1, 2006.

[18] S. Jiang, X. Li, S. Guo, Y. Hu, J. Yang, and Q. Jiang, "Performance of a piezoelectric bimorph for scavenging vibration energy," Smart Materials and Structures, vol. 14, no. 4, pp. 769-774, 2005.

[19] M. Ferrari, V. Ferrari, M. Guizzetti, B. Andò, S. Baglio, and C. Trigona, "Improved energy harvesting from wideband vibrations by nonlinear piezoelectric converters," Sensors and Actuators A: Physical, vol. 162, no. 2, pp. 425-431, 2010.

[20] G. J. Song, K.-B. Kim, J. Y. Cho et al., "Performance of a speed bump piezoelectric energy harvester for an automatic cellphone charging system," Applied Energy, vol. 247, pp. 221-227, 2019.

[21] Y. Wu, J. Qiu, S. Zhou, H. Ji, Y. Chen, and S. Li, "A piezoelectric spring pendulum oscillator used for multi-directional and ultra-low frequency vibration energy harvesting," Applied Energy, vol. 231, pp. 600-614, 2018.

[22] X. Bai, Y. Wen, P. Li, J. Yang, X. Peng, and X. Yue, "Multimodal vibration energy harvesting utilizing spiral cantilever with magnetic coupling," Sensors and Actuators A: Physical, vol. 209, pp. 78-86, 2014.

[23] J. L. Wang, G. P. Li, S. X. Zhou et al., "Enhancing wind energy harvesting using passive turbulence control devices," Applied Sciences, vol. 9, no. 5, p. 998, 2019.

[24] B. Marinkovic and H. Koser, "Smart sand-a wide bandwidth vibration energy harvesting platform," Applied Physics Letters, vol. 94, no. 10, Article ID 103505, 2009.

[25] M. Salauddin, R. M. Toyabur, P. Maharjan, and J. Y. Park, "High performance human-induced vibration driven hybrid energy harvester for powering portable electronics," Nano Energy, vol. 45, pp. 236-246, 2018. 
[26] K. Narendran, K. Murali, and V. Sundar, "Investigations into efficiency of vortex induced vibration hydro-kinetic energy device," Energy, vol. 109, pp. 224-235, 2016.

[27] H. Zhu and Y. Gao, "Hydrokinetic energy harvesting from flow-induced vibration of a circular cylinder with two symmetrical fin-shaped strips," Energy, vol. 165, pp. 1259$1281,2018$.

[28] R. Salazar, M. Serrano, and A. Abdelkefi, "Fatigue in piezoelectric ceramic vibrational energy harvesting: a review," Applied Energy, vol. 270, Article ID 115161, 2020.

[29] A. Hosseinkhani, D. Younesian, P. Eghbali, A. Moayedizadeh, and A. Fassih, "Sound and vibration energy harvesting for railway applications: a review on linear and nonlinear techniques," Energy Reports, vol. 7, pp. 852874, 2021.

[30] W. Hou, Y. Zheng, W. Guo, and P. Guo, "Piezoelectric vibration energy harvesting for rail transit bridge with steelspring floating slab track system," Journal of Cleaner Production, vol. 291, Article ID 125283, 2020.

[31] M. Derakhshani, N. Momenzadeh, and T. A. Berfield, "Analytical and experimental study of a clamped-clamped, bistable buckled beam low-frequency PVDF vibration energy harvester," Journal of Sound and Vibration, vol. 497, Article ID 115937, 2021.

[32] P. Kakou and O. Barry, "Simultaneous vibration reduction and energy harvesting of a nonlinear oscillator using a nonlinear electromagnetic vibration absorber-inerter," Mechanical Systems and Signal Processing, vol. 156, Article ID 107607, 2021.

[33] T. Yang, Q. J. Cao, and Z. F. Hao, "A novel nonlinear mechanical oscillator and its application in vibration isolation and energy harvesting," Mechanical Systems and Signal Processing, vol. 155, Article ID 107636, 2021.

[34] X. X. Wei, X. Liu, C. Y. Zheng et al., “A piezoelectric power generator based on axisymmetrically distributed PVDF array for two-dimension vibration energy harvesting and direction sensing," Sustainable Energy Technologies and Assessments, vol. 44, Article ID 101001, 2021.

[35] M. M. Bernitsas, K. Raghavan, Y. Ben-Simon et al., "VIVACE (vortex induced vibration aquatic clean energy): a new concept in generation of clean and renewable energy from fluid flow," Journal of Offshore Mechanics and Arctic Engineering, vol. 130, no. 4, Article ID 041101, 2008.

[36] R. D. Blevins, Flow-induced Vibration, Van Nostrand Reinhold, New York, NY, USA, 1990.

[37] H. J. Zhu, T. Tang, and J. L. Wang, "Modification of wake flow and air-bubble-vortex interference in the flow control of a circular cylinder with a pair of air jets: effect of injection velocity," Ocean Engineering, vol. 214, Article ID 107766, 2020.

[38] A. B. Rostami and M. Armandei, "Renewable energy harvesting by vortex-induced motions: review and benchmarking of technologies," Renewable and Sustainable Energy Reviews, vol. 70, pp. 193-214, 2017.

[39] A. Abdelkefi, F. Najar, A. H. Nayfeh et al., "An energy harvester using piezoelectric cantilever beams undergoing coupled bending-torsion vibrations," Smart Materials and Structures, vol. 20, no. 11, Article ID 115007, 2011.

[40] A. Bibo and M. F. Daqaq, "Investigation of concurrent energy harvesting from ambient vibrations and wind using a single piezoelectric generator," Applied Physics Letters, vol. 102, no. 24, Article ID 243904, 2013.

[41] A. Mehmood, A. Abdelkefi, M. R. Hajj et al., "Piezoelectric energy harvesting from vortex-induced vibrations of circular cylinder," Journal of Sound and Vibration, vol. 332, no. 13, pp. 4656-4667, 2013.

[42] Z. H. Lai, J. L. Wang, C. L. Zhang et al., "Harvest wind energy from a vibro-impact DEG embedded into a bluff body," Energy Conversion and Management, vol. 199, Article ID 111993, 2019.

[43] S. M. Shahruz, "Design of mechanical band-pass filters for energy scavenging," Journal of Sound and Vibration, vol. 292, no. 3-5, pp. 987-998, 2006.

[44] S. M. Shahruz, "Limits of performance of mechanical bandpass filters used in energy scavenging," Journal of Sound and Vibration, vol. 293, no. 1-2, pp. 449-461, 2006.

[45] Z. H. Lai, S. Wang, L. K. Zhu et al., "A hybrid piezo-dielectric wind energy harvester for high-performance vortex-induced vibration energy harvesting," Mechanical Systems and Signal Processing, vol. 150, Article ID 107212, 2021.

[46] K. Moon, J. Choe, H. Kim, D. Ahn, and J. Jeong, "A method of broadening the bandwidth by tuning the proof mass in a piezoelectric energy harvesting cantilever," Sensors and Actuators A: Physical, vol. 276, pp. 17-25, 2018.

[47] K. Yang, J. L. Wang, and D. Yurchenko, “A double-beam piezo-magneto-elastic wind energy harvester for improving the galloping-based energy harvesting," Applied Physics Letters, vol. 115, no. 19, Article ID 193901, 2019.

[48] R. Naseer, H. L. Dai, A. Abdelkefi, and L. Wang, "Piezomagnetoelastic energy harvesting from vortex-induced vibrations using monostable characteristics," Applied Energy, vol. 203, pp. 142-153, 2017.

[49] L. Y. Zhao and Y. W. Yang, "Enhanced aeroelastic energy harvesting with a beam stiffener," Smart Materials and Structures, vol. 24, Article ID 032001, 2015.

[50] L. Mateu and F. Moll, "Optimum piezoelectric bending beam structures for energy harvesting using shoe inserts," Journal of Intelligent Material Systems and Structures, vol. 16, no. 10, pp. 835-845, 2005.

[51] S. Roundy, E. S. Leland, J. Baker et al., "Improving power output for vibration-based energy scavengers," IEEE Pervasive Computing, vol. 4, no. 1, pp. 28-36, 2005.

[52] H. W. Kim, A. Batra, S. Priya et al., "Energy harvesting using a piezoelectric "cymbal" transducer in dynamic environment," Japanese Journal of Applied Physics, vol. 43, no. 9A, pp. 6178-6183, 2004.

[53] H. L. Dai, A. Abdelkefi, Y. Yang et al., "Orientation of bluff body for designing efficient energy harvesters from vortexinduced vibrations," Applied Physics Letters, vol. 108, no. 5, Article ID 053902, 2016.

[54] L. Ding, L. Zhang, M. M. Bernitsas, and C.-C. Chang, "Numerical simulation and experimental validation for energy harvesting of single-cylinder VIVACE converter with passive turbulence control," Renewable Energy, vol. 85, pp. 1246-1259, 2016.

[55] H. Park, R. A. Kumar, and M. M. Bernitsas, "Enhancement of flow-induced motion of rigid circular cylinder on springs by localized surface roughness at $3 \times 10^{4} \leq \mathrm{Re} \leq 1.2 \times 10^{5}$," Ocean Engineering, vol. 72, pp. 403-415, 2013.

[56] H. Zhu, Y. Gao, and T. Zhou, "Flow-induced vibration of a locally rough cylinder with two symmetrical strips attached on its surface: effect of the location and shape of strips," Applied Ocean Research, vol. 72, pp. 122-140, 2018.

[57] G. Hu, K. T. Tse, K. C. S. Kwok et al., "Aerodynamic modification to a circular cylinder to enhance the piezoelectric wind energy harvesting," Applied Physics Letters, vol. 109, no. 19, Article ID 193902, 2016. 
[58] J. Wang, G. Zhao, M. Zhang, and Z. Zhang, "Efficient study of a coarse structure number on the bluff body during the harvesting of wind energy," Energy Sources, Part A: Recovery, Utilization, and Environmental Effects, vol. 40, no. 15, pp. 1788-1797, 2018.

[59] G. Hu, K. T. Tse, M. H. Wei et al., "Experimental investigation on the efficiency of circular cylinder-based wind energy harvester with different rod-shaped attachments," Applied Energy, vol. 226, pp. 628-689, 2018.

[60] G. Hu, K. T. Tse, and K. C. S. Kwok, "Enhanced performance of wind energy harvester by aerodynamic treatment of a square prism," Applied Physics Letters, vol. 108, no. 12, Article ID 123901, 2016.

[61] J. Song, G. Hu, K. T. Tse et al., "Performance of a circular cylinder piezoelectric wind energy harvester fitted with a splitter plate," Applied Physics Letters, vol. 111, no. 22, Article ID 223903, 2017.

[62] H. J. Zhu, G. M. Li, and J. L. Wang, "Flow-induced vibration of a circular cylinder with splitter plates placed upstream and downstream individually and simultaneously," Applied Ocean Research, vol. 97, Article ID 102084, 2020.

[63] H. J. Zhu and W. L. Liu, "Flow control and vibration response of a circular cylinder attached with a wavy plate," Ocean Engineering, vol. 212, Article ID 105737, 2020.

[64] J. Wang, S. Zhou, Z. Zhang, and D. Yurchenko, "Highperformance piezoelectric wind energy harvester with $Y$ shaped attachments," Energy Conversion and Management, vol. 181, pp. 645-652, 2019.

[65] H. J. Zhu and T. M. Zhou, "Flow around a circular cylinder attached with a pair of fin-shaped strips," Ocean Engineering, vol. 190, Article ID 106484, 2019.

[66] H. J. Zhu, W. L. Liu, and T. M. Zhou, "Direct numerical simulation of the wake adjustment and hydrodynamic characteristics of a circular cylinder symmetrically attached with fin-shaped strips," Ocean Engineering, vol. 195, Article ID 106756, 2020.

[67] L. Ding, L. Yang, Z. M. Yang et al., "Performance improvement of aeroelastic energy harvesters with two symmetrical fin-shaped rods," Journal of Wind Engineering and Industrial Aerodynamics, vol. 196, Article ID 104051, 2020.

[68] C. Ma, H. Sun, G. Nowakowski, E. Mauer, and M. M. Bernitsas, "Nonlinear piecewise restoring force in hydrokinetic power conversion using flow induced motions of single cylinder," Ocean Engineering, vol. 128, pp. 1-12, 2016.

[69] J. H. Lee and M. M. Bernitsas, "High-damping, high-Reynolds VIV tests for energy harnessing using the VIVACE converter," Ocean Engineering, vol. 38, no. 16, pp. 1697-1712, 2011.

[70] G. Hu, F. X. Liu, C. Li et al., "Wind energy harvesting performance of tandem circular cylinders with triangular protrusions," Journal of Fluids and Structures, vol. 91, Article ID 102780, 2019.

[71] S. Kang, S. Ryu, and S. Song, "Quantification of VIV-driven energy transfer for a circular cylinder with a pair of bumps at Reynolds number 150," Journal of Mechanical Science and Technology, vol. 34, no. 3, pp. 1139-1147, 2020.

[72] H. L. Dai, A. Abdelkefi, and L. Wang, "Piezoelectric energy harvesting from concurrent vortex-induced vibrations and base excitations," Nonlinear Dynamics, vol. 77, no. 3, pp. 967-981, 2014.

[73] H. Dai, A. Abdelkefi, and L. Wang, "Theoretical modeling and nonlinear analysis of piezoelectric energy harvesting from vortex-induced vibrations," Journal of Intelligent
Material Systems and Structures, vol. 25, no. 14, pp. 18611874, 2014.

[74] H. D. Akaydin, N. Elvin, and Y. Andreopoulos, "The performance of a self-excited fluidic energy harvester," Smart Materials and Structures, vol. 21, no. 2, Article ID 025007, 2012.

[75] J. D. Jia, X. B. Shan, D. Upadrashta et al., "An asymmetric bending-torsional piezoelectric energy harvester at low wind speed," Energy, vol. 198, Article ID 117287, 2020.

[76] F. Ewere, G. Wang, and B. Cain, "Experimental investigation of galloping piezoelectric energy harvesters with square bluff bodies," Smart Materials and Structures, vol. 23, no. 10, Article ID 104012, 2014.

[77] M. Zhang, G. F. Zhao, and J. L. Wang, "Study on fluidinduced vibration power harvesting of square columns under different attack angles," Geofluids, vol. 2017, Article ID 6439401, 18 pages, 2017.

[78] J. Wang, L. Tang, L. Zhao, and Z. Zhang, "Efficiency investigation on energy harvesting from airflows in HVAC system based on galloping of isosceles triangle sectioned bluff bodies," Energy, vol. 172, pp. 1066-1078, 2019.

[79] H. J. Zhu, T. Tang, T. M. Zhou et al., "Flow structures around trapezoidal cylinders and their hydrodynamic characteristics: effect of the base length ratio and attack angle," Physics of Fluids, vol. 32, Article ID 103606, 2020.

[80] J. Sirohi and R. Mahadik, "Harvesting wind energy using a galloping piezoelectric beam," Journal of Vibration and Acoustics, vol. 134, no. 1, Article ID 011009, 2012.

[81] S. D. Kwon, "A T-shaped piezoelectric cantilever for fluid energy harvesting," Applied Physics Letters, vol. 97, no. 16, Article ID 164102, 2010.

[82] F. R. Liu, H. X. Zou, W. M. Zhang et al., "Y-type three-blade bluff body for wind energy harvesting," Applied Physics Letters, vol. 112, no. 23, Article ID 233903, 2018.

[83] F.-R. Liu, W.-M. Zhang, Z.-K. Peng, and G. Meng, "Forkshaped bluff body for enhancing the performance of galloping-based wind energy harvester," Energy, vol. 183, pp. 92-105, 2019.

[84] F. Ewere, G. Wang, and A. Frendi, "Experimental investigation of a bioinspired bluff-body effect on galloping piezoelectric energy-harvester performance," AIAA Journal, vol. 56, no. 3, pp. 1284-1287, 2018.

[85] J. L. Wang, S. H. Gu, C. Y. Zhang et al., "Hybrid wind energy scavenging by coupling vortex-induced vibrations and galloping," Energy Conversion and Management, vol. 213, Article ID 112835, 2020.

[86] A. Abdelkefi, Z. Yan, and M. R. Hajj, "Performance analysis of galloping-based piezoaeroelastic energy harvesters with different cross-section geometries," Journal of Intelligent Material Systems and Structures, vol. 25, no. 2, pp. 246-256, 2014.

[87] J. M. Kluger, F. C. Moon, and R. H. Rand, "Shape optimization of a blunt body vibro-wind galloping oscillator," Journal of Fluids and Structures, vol. 40, pp. 185-200, 2013.

[88] K. Gkoumas, F. Petrini, and F. Bontempi, "Piezoelectric vibration energy harvesting from airflow in HVAC (heating ventilation and air conditioning) systems," Procedia Engineering, vol. 199, pp. 3444-3449, 2017.

[89] W. P. Sun, D. L. Zhao, T. Tan et al., "Low velocity water flow energy harvesting using vortex induced vibration and galloping," Applied Energy, vol. 251, Article ID 113392, 2019.

[90] Y. W. Yang, L. Y. Zhao, and L. H. Tang, "Comparative study of tip cross-sections for efficient galloping energy 
harvesting," Applied Physics Letters, vol. 102, no. 6, Article ID 064105, 2013.

[91] H. Wang, Q. Zhai, and K. Chen, "Vortex-induced vibrations of an elliptic cylinder with both transverse and rotational degrees of freedom," Journal of Fluids and Structures, vol. 84, pp. 36-55, 2019.

[92] L. Lu, Z. L. Guo, G. Q. Tang et al., "Numerical investigation of flow-induced rotary oscillation of a circular cylinder with rigid splitter plate," Physics of Fluids, vol. 28, Article ID 093604, 2016.

[93] H. J. Zhu, Y. Zhao, and J. Hu, "Performance of a novel energy harvester for energy self-sufficiency as well as a vortex-induced vibration suppressor," Journal of Fluids and Structures, vol. 91, Article ID 102736, 2019.

[94] H. Zhu and Y. Gao, "Vortex induced vibration response and energy harvesting of a marine riser attached by a free-torotate impeller," Energy, vol. 134, pp. 532-544, 2017.

[95] U. Javed and A. Abdelkefi, "Role of the galloping force and moment of inertia of inclined square cylinders on the performance of hybrid galloping energy harvesters," Applied Energy, vol. 231, pp. 259-276, 2018.

[96] H. Sun and M. M. Bernitsas, "Bio-Inspired adaptive damping in hydrokinetic energy harnessing using flow-induced oscillations," Energy, vol. 176, pp. 940-960, 2019.

[97] L. B. Zhang, B. Meng, Y. Xia et al., "Galloping triboelectric nanogenerator for energy harvesting under low wind speed," Nano Energy, vol. 70, Article ID 104477, 2020.

[98] H. Zhu, Y. Zhao, and T. Zhou, "CFD analysis of energy harvesting from flow induced vibration of a circular cylinder with an attached free-to-rotate pentagram impeller," Applied Energy, vol. 212, pp. 304-321, 2018.

[99] M. Usman, A. Hanif, I.-H. Kim, and H.-J. Jung, "Experimental validation of a novel piezoelectric energy harvesting system employing wake galloping phenomenon for a broad wind spectrum,” Energy, vol. 153, pp. 882-889, 2018.

[100] A. H. Alhadidi and M. F. Daqaq, "A broadband bi-stable flow energy harvester based on the wake-galloping phenomenon," Applied Physics Letters, vol. 109, no. 3, Article ID 033904, 2016.

[101] J. L. Wang, L. F. Geng, M. Zhang et al., "Broadening band of wind speed for aeroelastic energy scavenging of a cylinder through buffeting in the wake of a square prism," Shock and Vibration, vol. 2018, Article ID 2039561, 14 pages, 2018.

[102] M. Rezaei and R. Talebitooti, "Wideband PZT energy harvesting from the wake of a bluff body in varying flow speeds," International Journal of Mechanical Sciences, vol. 163, Article ID 105135, 2019.

[103] Y. Hu, B. Yang, X. Chen, X. Wang, and J. Liu, "Modeling and experimental study of a piezoelectric energy harvester from vortex shedding-induced vibration," Energy Conversion and Management, vol. 162, pp. 145-158, 2018.

[104] L. A. Weinstein, M. R. Cacan, P. M. So et al., "Vortex shedding induced energy harvesting form piezoelectric materials in heating, ventilation and air conditioning flows," Smart Materials and Structures, vol. 21, Article ID 045003, 2012.

[105] H. D. Akaydm, N. Elvin, and Y. Andreopoulos, "Wake of a cylinder: a paradigm for energy harvesting with piezoelectric materials," Experiments in Fluids, vol. 49, no. 1, pp. 291-304, 2010.

[106] O. Goushcha, N. Elvin, and Y. Andreopoulos, "Interactions of vortices with a flexible beam with applications in fluidic energy harvesting," Applied Physics Letters, vol. 104, no. 2, Article ID 021919, 2014.
[107] X. Shan, R. Song, B. Liu, and T. Xie, "Novel energy harvesting: a macro fiber composite piezoelectric energy harvester in the water vortex," Ceramics International, vol. 41, pp. S763-S767, 2015.

[108] H. J. Zhu and K. N. Wang, "Wake adjustment and vortexinduced vibration of a circular cylinder with a C-shaped plate at a low Reynolds number of 100," Physics of Fluids, vol. 31, Article ID 103602, 2019.

[109] H. J. Zhu, C. Zhang, and W. L. Liu, "Wake-induced vibration of a circular cylinder at a low Reynolds number of 100," Physics of Fluids, vol. 31, Article ID 073606, 2019.

[110] H. J. Zhu, X. N. Tan, Y. Gao et al., "Two-degree-of-freedom flow-induced vibration of two rigidly coupled tandem cylinders of unequal diameters," Ocean Engineering, vol. 216, Article ID 108142, 2020.

[111] A. Abdelkefi, J. M. Scanlon, E. McDowell et al., "Performance enhancement of piezoelectric energy harvesters from wake galloping," Applied Physics Letters, vol. 103, no. 3, Article ID 033903, 2013.

[112] G. Hu, J. L. Wang, Z. Su et al., "Performance evaluation of twin piezoelectric wind energy harvesters under mutual interference," Applied Physics Letters, vol. 115, no. 7, Article ID 073901, 2019.

[113] X. Shan, H. Li, Y. Yang, J. Feng, Y. Wang, and T. Xie, "Enhancing the performance of an underwater piezoelectric energy harvester based on flow-induced vibration," Energy, vol. 172, pp. 134-140, 2019.

[114] S. X. Zhou and J. L. Wang, "Dual serial vortex-induced energy harvesting system for enhanced energy harvesting," AIP Advances, vol. 8, no. 7, Article ID 075221, 2018.

[115] W. B. Hobbs and D. L. Hu, "Tree-inspired piezoelectric energy harvesting," Journal of Fluids and Structures, vol. 28, pp. 103-114, 2012.

[116] L. B. Zhang, H. L. Dai, A. Abdelkefi et al., "Improving the performance of aeroelastic energy harvesters by an interference cylinder," Applied Physics Letters, vol. 111, no. 7, Article ID 073904, 2017.

[117] L. B. Zhang, H. L. Dai, A. Abdelkefi, and L. Wang, "Experimental investigation of aerodynamic energy harvester with different interference cylinder cross-sections," Energy, vol. 167, pp. 970-981, 2019.

[118] W. Sun, F. Guo, and J. Seok, "Development of a novel vibrowind galloping energy harvester with high power density incorporated with a nested bluff-body structure," Energy Conversion and Management, vol. 197, Article ID 111880, 2019.

[119] L. Y. Zhao, L. H. Tang, and Y. W. Yang, "Enhanced piezoelectric galloping energy harvesting using 2 degree-offreedom cut-out cantilever with magnetic interaction," Japanese Journal of Applied Physics, vol. 53, no. 6, Article ID 060302, 2014.

[120] R. Song, X. Shan, F. Lv, J. Li, and T. Xie, "A novel piezoelectric energy harvester using the macro fiber composite cantilever with a bicylinder in water," Applied Sciences, vol. 5, no. 4, pp. 1942-1954, 2015.

[121] J. L. Wang, G. B. Hu, Z. Su et al., "A cross-coupled dual-beam for multidirectional energy harvesting from vortex induced vibrations," Smart Materials and Structures, vol. 28, no. 12, Article ID 12LT02, 2019.

[122] H. Vocca, I. Neri, F. Travasso, and L. Gammaitoni, "Kinetic energy harvesting with bistable oscillators," Applied Energy, vol. 97, pp. 771-776, 2012.

[123] L. B. Zhang, A. Abdelkefi, H. L. Dai, R. Naseer, and L. Wang, "Design and experimental analysis of broadband energy 
harvesting from vortex-induced vibrations," Journal of Sound and Vibration, vol. 408, pp. 210-219, 2017.

[124] J. Wang, L. Geng, K. Yang, L. Zhao, F. Wang, and D. Yurchenko, "Dynamics of the double-beam piezo-magneto-elastic nonlinear wind energy harvester exhibiting galloping-based vibration," Nonlinear Dynamics, vol. 100, no. 3, pp. 1963-1983, 2020.

[125] S. Zhou, J. Cao, D. J. Inman, J. Lin, S. Liu, and Z. Wang, "Broadband tristable energy harvester: modeling and experiment verification," Applied Energy, vol. 133, pp. 33-39, 2014.

[126] J. Wang, L. Geng, S. Zhou, Z. Zhang, Z. Lai, and D. Yurchenko, "Design, modeling and experiments of broadband tristable galloping piezoelectric energy harvester," Acta Mechanica Sinica, vol. 36, no. 3, pp. 592-605, 2020. 